\title{
DIFFERENCE OPERATORS FROM INTERPOLATING MOVING LEAST SQUARES AND THEIR DEVIATION FROM OPTIMALITY
}

\author{
THOMAS SONAR ${ }^{1}$
}

\begin{abstract}
We consider the classical Interpolating Moving Least Squares (IMLS) interpolant as defined by Lancaster and Šalkauskas [Math. Comp. 37 (1981) 141-158] and compute the first and second derivative of this interpolant at the nodes of a given grid with the help of a basic lemma on Shepard interpolants. We compare the difference formulae with those defining optimal finite difference methods and discuss their deviation from optimality.
\end{abstract}

Mathematics Subject Classification. 39A70, 39A12, 65D05, 65D25.

Received: August 31, 2004.

\section{INTRODUCTION}

During the last decade several meshless methods for the solution of partial differential equations were developed, among them particle methods, finite element-type methods and methods of generalized finite differences, see [1] for a review of the state-of-the-art in the midst 1990s. Although the history of finite difference-based meshless methods started as far back as 1975 with the classical paper by Perrone and Kao [11] which was itself taken up by Liszka and Orkisz in [9] the development in this area is by far not as fast as in other classes of meshless methods. There are several reasons for this situation. First of all finite elements provide a versatile machinery for problems of (seemingly) all kind because the theory is completely imbedded in mathematical analysis. In contrast, finite differences are really discrete operators and even the link to the continuous operators which they represent is only possible by exploiting Taylor series arguments - a fairly low-level weapon if compared to the finite element theories where the "discrete" spaces are itself continuous and imbedded in beautiful function spaces. As an implication of the aforesaid much more researchers have turned their attention towards finite element methods but in classical areas of finite differences like numerical fluid dynamics many people moved into finite volume techniques which are finite difference methods on irregular grids. Hence, due to the simplicity and versatility of finite differences in applications there is a need for meshless finite difference methods. Fortunately, there always have been a few researchers interested in the theory and applications of "meshless" finite differences. In Germany we find early work from 1982 in a Ph.D. Thesis of an aeronautical engineer from the University of Stuttgart with the title Ein gitterfreies Differenzenverfahren (A meshfree difference method) [13], in which flow around turbine blades was analyzed with the help of a meshless finite difference method. While the method used (based on Taylor series expansions, of course) depended strongly on a certain regularity in the point distribution it was Schönauer and his co-workers who developed practically

Keywords and phrases. Difference operators, moving least squares interpolation, order of approximation.

${ }^{1}$ Institut Computational Mathematics, TU Braunschweig, Pockelsstraße 14, 38106 Braunschweig, Germany. t.sonar@tu-bs.de

(c) EDP Sciences, SMAI 2005 
relevant algorithms to choose stable finite differences on points of clouds but who also established the first links to difference operators in order to analyze the quality of his meshless derivatives, see [12]. Coming from a different direction Fürst and the author constructed in [5] a meshless finite difference scheme on the basis of John's theory of positive schemes for parabolic equations.

In contrast to using locally defined differences derived by Taylor series expansions another approach is given by the classical moving least squares (MLS) method as derived by Lancaster and Šalkauskas in [7]. Here, a globally defined meshless approximant is used in order to compute derivatives at certain nodes. Many authors were concerned with the approximating moving least squares (AMLS) method but only few used the interpolating MLS (IMLS) method since this technique leads to singular matrices and can be handled numerically with great care only. It was another Ph.D. thesis [6] in which the IMLS technique was used in order to compute compressible flow problems in one space dimension. In this thesis the IMLS interpolants are derived differently from [7] by an ingenious asymptotic argument in matrix computation and the IMLS is compared to the AMLS by looking at numerical examples.

The present work starts a series of three papers which are concerned with the analysis of the IMLS method and its interpretation as a generalized ("meshless") finite difference method. In this paper the IMLS method is constructed exactly as in [7] in Section 2. From this construction we prove a useful Theorem on the derivatives of the Shepard interpolant which is the "building block" of the method. We then construct IMLS interpolants based on a linear as well as on a quadratic polynomial basis and document their performance with numerical experiments. After recalling optimal finite difference formulae in Section 3 we start a detailed analysis of IMLS interpolants in Section 4. It is here where exact formulae for the first and second derivatives of IMLS interpolants at the nodes are derived. It is shown that the second derivative of an IMLS interpolant based on a linear polynomial basis is inconsistent. In Section 5 we establish the link between IMLS interpolants and finite difference operators in which we compute first and second differences from the interpolant on small sets of points i.e. under a restriction on the support of certain weight functions. Besides some structural insight it turns out that the IMLS interpolants yield optimal formulae by restricting the support of the weights but lead to sub-optimal formulae in case of unrestricted supports. Although these results may seem to yield arguments against the use of IMLS interpolants in the numerical computation of partial differential equations they may turn out to be quite useful in terms of stability. This, however, is one of the topics of the following two papers from which both already exist in manuscript, $[10,14]$, and will be submitted soon.

In that second paper the Ansatz of Kunle [6] is taken up and analyzed in a multidimensional setting. It will be shown that it is versatile enough to solve problems in compressible fluid mechanics as well as the incompressible Navier-Stokes equations in two space dimensions. In a third and final paper [14] we shall consider stability properties of the difference operators derived.

\section{INTERPOLATING MOVING LEAST SQUARES}

The moving least squares (MLS) technique for interpolation derives all its advantages as well as its disadvantages directly from the idea of construction: for given data of a function $f: D \subset \mathbf{R} \rightarrow \mathbf{R}$ and a fixed point $\tilde{x} \in D$ one looks for a function

$$
L_{\tilde{x}} f(x):=\sum_{i=1}^{n} a_{i}(\tilde{x}) b^{(i)}(x)
$$

expanded with respect to a given basis $\left\{b^{(0)}, b^{(0)}, \ldots, b^{(n)}\right\}$ which is the best approximation to $f$ in a certain least squares sense (for details see [7]). Then this local approximation is shifted over $D$ and hence a global function

$$
G f(\tilde{x})=L_{\tilde{x}} f(\tilde{x})=\sum_{i=1}^{n} a_{i}(\tilde{x}) b^{(i)}(\tilde{x})
$$

is achieved. If we assume that data

$$
\left(x_{1}, f_{1}\right), \ldots,\left(x_{N}, f_{N}\right)
$$


with $f_{k}:=f\left(x_{k}\right)$ is given on a grid we may introduce different weights to different points by defining functions

$$
w^{(i)}(x):=w\left(x-x_{i}\right)
$$

Following Lancaster and Šalkauskas the computation of a least square function in the sense described above requires the solution of coefficients $\boldsymbol{a}^{T}(x):=\left(a_{1}(x), \ldots, a_{N}(x)\right)$ from the normal equations

$$
\boldsymbol{B} \boldsymbol{W}(x) \boldsymbol{B}^{\top} \boldsymbol{a}=\boldsymbol{B} \boldsymbol{W}(x) \boldsymbol{f}
$$

where $\boldsymbol{f}^{T}:=\left(f_{1}, \ldots, f_{N}\right)$ and $\boldsymbol{B}:=\left(\begin{array}{ccc}b^{(1)}\left(x_{1}\right) & \cdots & b^{(1)}\left(x_{N}\right) \\ \vdots & \ddots & \vdots \\ b^{(n)}\left(x_{1}\right) & \cdots & b^{(n)}\left(x_{N}\right)\end{array}\right)$ and $\boldsymbol{W}(x):=\operatorname{diag}\left(w^{(1)}(x), \ldots, w^{(N)}(x)\right)$. We shall confine ourselves to the linear and quadratic polynomial basis

$$
\left\{b^{(1)}(x)=1, b^{(2)}(x)=x\right\}
$$

and

$$
\left\{b^{(1)}(x)=1, b^{(2)}(x)=x, b^{(3)}(x)=x^{2}\right\},
$$

respectively. It is easily seen that in order to interpolate the given data weights with $\lim _{x \rightarrow x_{i}} w^{(i)}(x)=\infty$ are necessary. In this paper we will always use the function classes

$$
w^{(i)}(x):=\frac{1}{\left(x-x_{i}\right)^{\alpha}}, \quad \alpha \text { even. }
$$

Note that the choice of the weights is completely independent from the choice of the basis functions. As can be seen from the normal equations the coefficients of the interpolating moving least squares (IMLS) function $G f$ can not be computed once on a discrete set of points as in the case of polynomial interpolation. In contrast, the coefficients have to be computed at every point where the value of $G f$ is needed. Moreover, at the nodes $x_{k}$ there is a singularity occurring in $\boldsymbol{W}$ making it impossible to compute the coefficients directly from the normal equations in general. However, the case $n=1$ corresponds to the Shepard interpolant and can be treated directly.

Due to our choice of weight functions the sum $\sum_{i=1}^{N} w^{(i)}(x)$ is positive. Hence, dividing the normal equations (1) by this sum does not change the solution. We arrive at

$$
\boldsymbol{B} \boldsymbol{V}(x) \boldsymbol{B}^{\top} \boldsymbol{a}(x)=\boldsymbol{B} \boldsymbol{V}(z) \boldsymbol{f}
$$

with $\boldsymbol{V}(x)=\operatorname{diag}\left(v^{(1)}(x), \ldots, v^{(N)}(x)\right)$ and

$$
v^{(i)}(x):=\frac{w^{(i)}(x)}{\sum_{j=1}^{N} w^{(j)}(x)} .
$$

It is easily seen that the weight functions $v^{(i)}$ enjoy the following properties:

(1) $v^{(i)}\left(x_{j}\right)=\delta_{j}^{i}$

(2) $0 \leq v^{(i)}(x) \leq 1$ for all $x \in D$;

(3) $\sum_{i=1}^{N} v^{(i)}(x)=1$; and

(4) $v^{(i)}=\mathcal{O}(1 / N)$ for $|x| \rightarrow \infty$.

In case $n=1$ i.e. the basis $\left\{b^{(1)}(x)=1\right\}$, the normal equations (5) give immediately

$$
\left(\sum_{i=1}^{N} v^{(i)}(x)\right) a_{1}(x)=\sum_{i=1}^{N} f_{i} v^{(i)}(x)
$$


and since $\sum_{i=1}^{N} v^{(i)}(x)=1$ and $b^{(1)}(x) \equiv 1$ this results in

$$
S f(x):=G f(x)=\sum_{i=1}^{N} f_{i} v^{(i)}(x)
$$

which is called the Shepard interpolant.

Remark 2.1. We note in passing that in their book [8] Lancaster and Šalkauskas erroneously describe the computation of first and second derivatives at the nodes of an arbitrary IMLS interpolant based on the weights $v^{(i)}$. The presentation given there (p. 233) is based on the equation $\boldsymbol{B} \boldsymbol{V}(x) \boldsymbol{B}^{\top} \frac{\mathrm{d}}{\mathrm{d} x} \boldsymbol{a}(x)=\boldsymbol{B} \frac{\mathrm{d}}{\mathrm{d} x} \boldsymbol{V}(x)\left(\boldsymbol{f}-\boldsymbol{B}^{\top} \boldsymbol{a}\right)$ and the observation that at every node $x_{k}$ the matrix $\frac{\mathrm{d}}{\mathrm{d} x} \boldsymbol{V}(x)$ will vanish due to the properties of the weights. The authors then conclude that $\frac{\mathrm{d}}{\mathrm{d} x} \boldsymbol{a}(x)$ will also vanish at the nodes. This, however, can not be concluded since the matrix $\boldsymbol{B} \boldsymbol{V}(x) \boldsymbol{B}^{\top}$ is of rank one at each node!

In [7] Lancaster and Šalkauskas used the Shepard interpolant as building block and succeeded in shifting the problems with the singularity in $\boldsymbol{W}(x)$ solely on $S f$. For reasons of easy reference let us give a summary of their Theorem 4.1:

Theorem 2.1. Let $b^{(i)} \in C^{m}(D), i=1, \ldots, n$, be a given basis and let the weights be given as in (4). Then the IMLS interpolant is given by

$$
G f(x)=S f(x)+\sum_{i=2}^{n} \beta_{i-1}(x) g^{(i)}(x)
$$

where

$$
g^{(i)}(x):=b^{(i)}(x)-S b^{(i)}(x)
$$

and $\boldsymbol{\beta}^{\top}(x):=\left(\beta_{1}(x), \ldots, \beta_{n-1}(x)\right)$ is solution of the linear system

$$
\boldsymbol{U}(x) \boldsymbol{W}(x) \boldsymbol{U}^{\top}(x) \boldsymbol{\beta}(x)=\boldsymbol{U} \boldsymbol{W}\left(\boldsymbol{f}-S f(x) \boldsymbol{b}^{(1)}\right)
$$

where $\boldsymbol{b}^{(1)}:=\left(b^{(1)}\left(x_{1}\right), \ldots, b^{(1)}\left(x_{N}\right)\right)^{\top}, \boldsymbol{U} \in \mathbf{R}^{(n-1) \times N}$ with row $(i-1)$ given by

$$
\begin{gathered}
\boldsymbol{u}^{(i)^{\top}}(x):=\left(u^{(i)}\left(x, x_{1}\right), \ldots, u^{(i)}\left(x, x_{N}\right)\right) \text { and } \\
u^{(i)}\left(x, x_{k}\right):=b^{(i)}\left(x_{k}\right)-S b^{(i)}(x), \quad i=2,3, \ldots, n .
\end{gathered}
$$

\subsection{The Shepard interpolant}

The case $n=1$ corresponds to the use of the constant basis and the interpolant was given in (6). The actual computation of the weights $v^{(i)}$ has to be done with some care due to the singularity in $w^{(i)}$ at a node. We recommend the use of

$$
v^{(i)}(x)=\frac{1}{\frac{w^{(1)}(x)}{w^{(i)}(x)}+\cdots+\frac{w^{(i-1)}(x)}{w^{(i)}(x)}+1+\frac{w^{(i+1)}(x)}{w^{(i)}(x)}+\cdots+\frac{w^{(N)}(x)}{w^{(i)}(x)}}
$$

and evaluated $v^{(i)}(x)$ for all $x$ with $\left|x-x_{i}\right|>10^{-12}$ in double precision arithmetic. In case of $\left|x-x_{i}\right| \leq 10^{-12}$ simply the value 1 was returned by the weight function.

We start with the function $f:[0,2 \pi] \rightarrow[0,2 \pi], x \mapsto x$ and use 10 equally spaced data points $0=x_{1}<x_{2}<\ldots<x_{N}=x_{10}=2 \pi$. The Shepard interpolant was then evaluated at 200 equally spaced data points $0=x_{1}^{\text {plot }}<x_{2}^{\text {plot }}<\ldots<x_{200}^{\text {plot }}=2 \pi$ and the Shepard function was then plotted as piecewise linear polygonal line. 


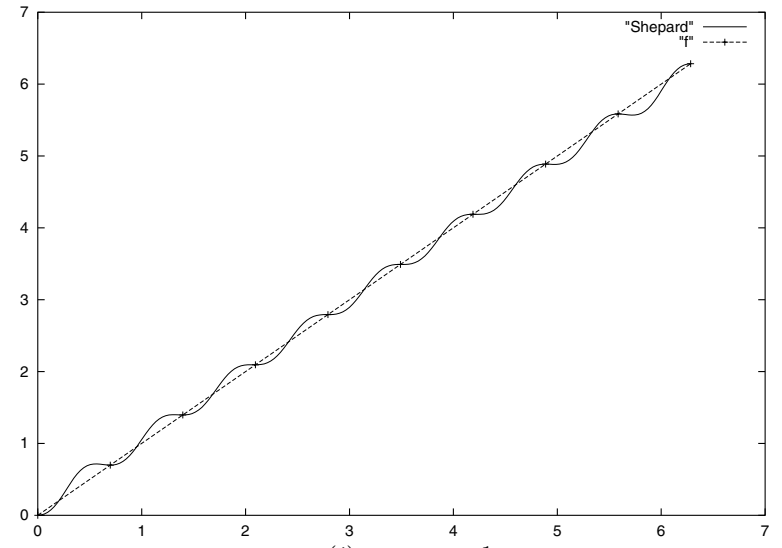

(a) $w^{(i)}(x)=\frac{1}{\left(x-x_{i}\right)^{2}}$

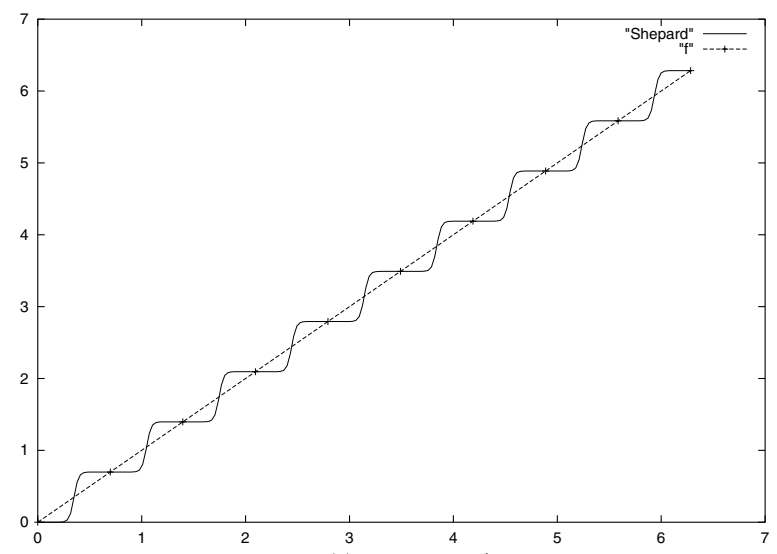

(c) $w^{(i)}(x)=\frac{1}{\left(x-x_{i}\right)^{8}}$

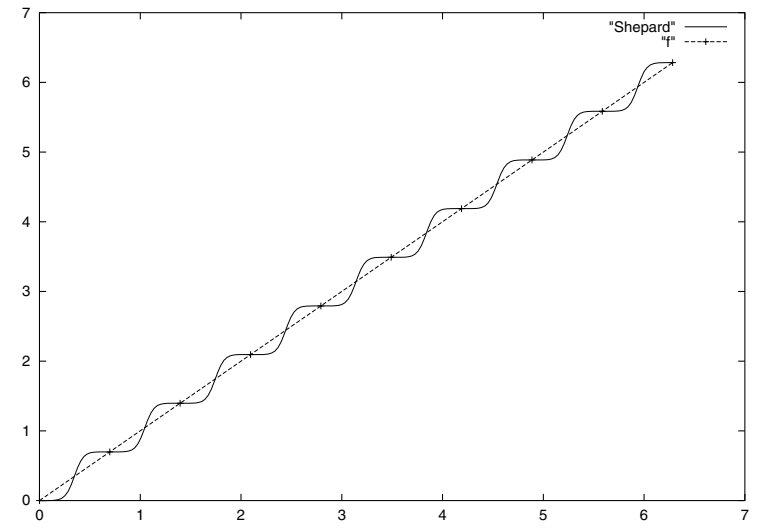

(b) $w^{(i)}(x)=\frac{1}{\left(x-x_{i}\right)^{4}}$

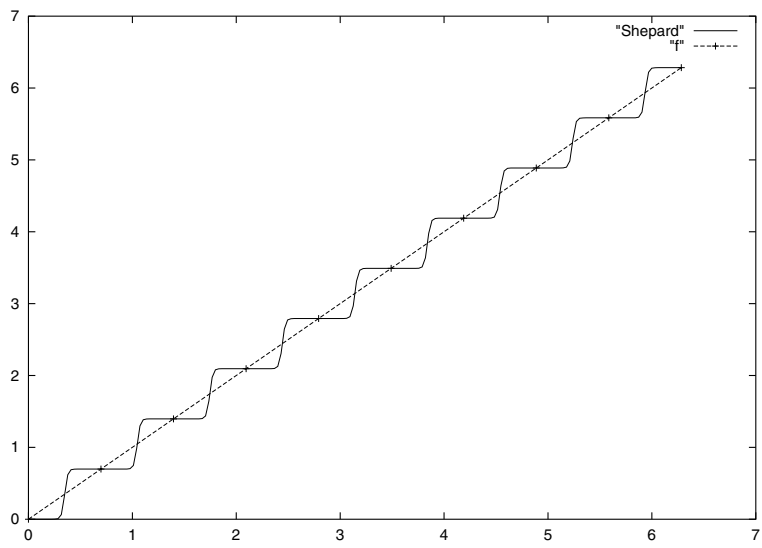

(d) $w^{(i)}(x)=\frac{1}{\left(x-x_{i}\right)^{12}}$

FIGURE 1. Shepard interpolants of $f(x)=x$ with four different weight functions.

The typical behaviour of the Shepard interpolant at the nodes is the so called flat spot phenomenon. It follows easily from the properties 1 and 2 of the weights $v^{(i)}$ listed above that

$$
\frac{\mathrm{d}}{\mathrm{d} x} S f\left(x_{k}\right)=0
$$

as is well known, compare [8]. We will come back to this flat spot phenomenon later and prove a useful lemma concerning the values of higher derivatives in connection with the weight function used.

It can be seen from the plots given in Figure 1 that for increasing singularity in the weights $w^{(i)}$ the Shepard interpolant tends towards a piecewise constant interpolation.

As a second example we consider interpolation of $f:[0,2 \pi] \rightarrow[-1,1], x \mapsto \sin x$. We use 20 equally spaced data points $0=x_{1}<x_{2}<\ldots<x_{N}=x_{20}=2 \pi$. The Shepard interpolant was again evaluated at 200 equally spaced data points $0=x_{1}^{\text {plot }}<x_{2}^{\text {plot }}<\ldots<x_{200}^{\text {plot }}=2 \pi$ and the Shepard function was plotted as piecewise linear polygonal line.

Obviously the flat spot phenomenon rules out the Shepard interpolant for purposes of the computation of derivatives. Enriched bases are needed to overcome the difficulties. 


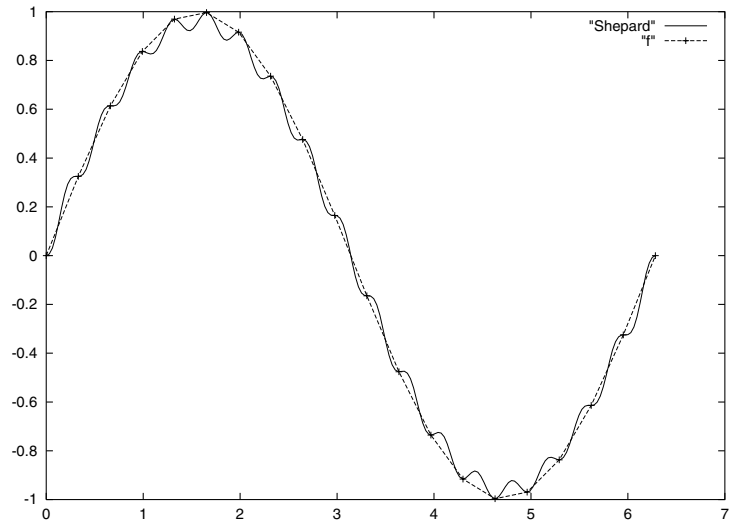

(a) $w^{(i)}(x)=\frac{1}{\left(x-x_{i}\right)^{2}}$

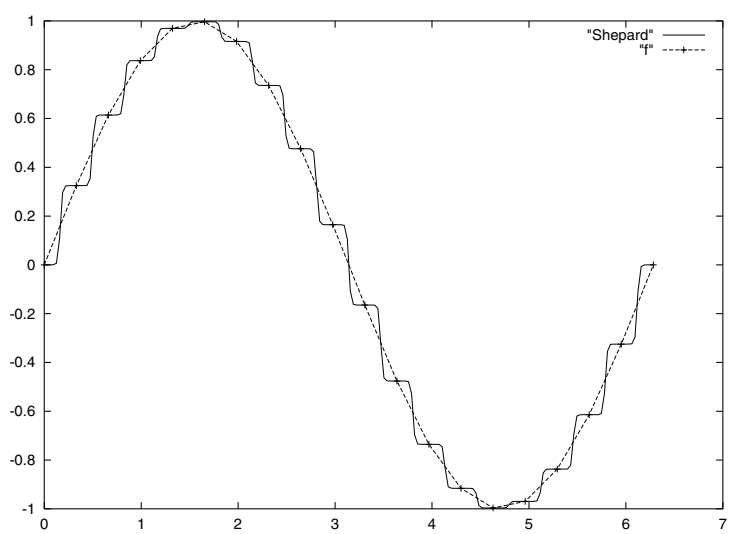

(c) $w^{(i)}(x)=\frac{1}{\left(x-x_{i}\right)^{8}}$

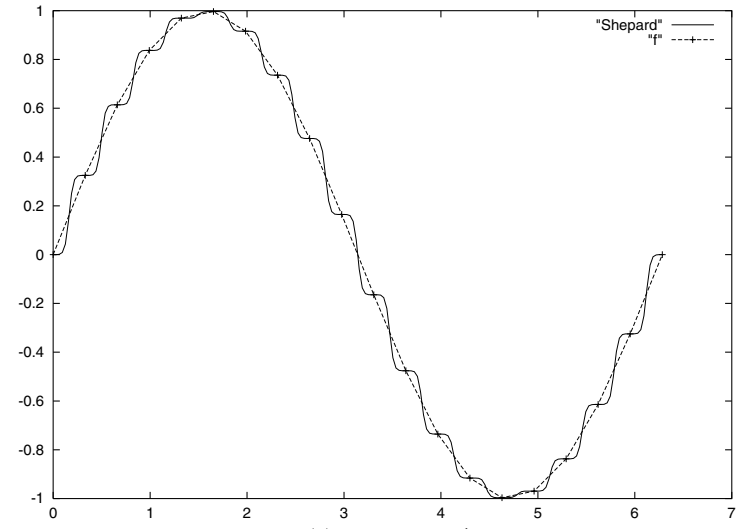

(b) $w^{(i)}(x)=\frac{1}{\left(x-x_{i}\right)^{4}}$

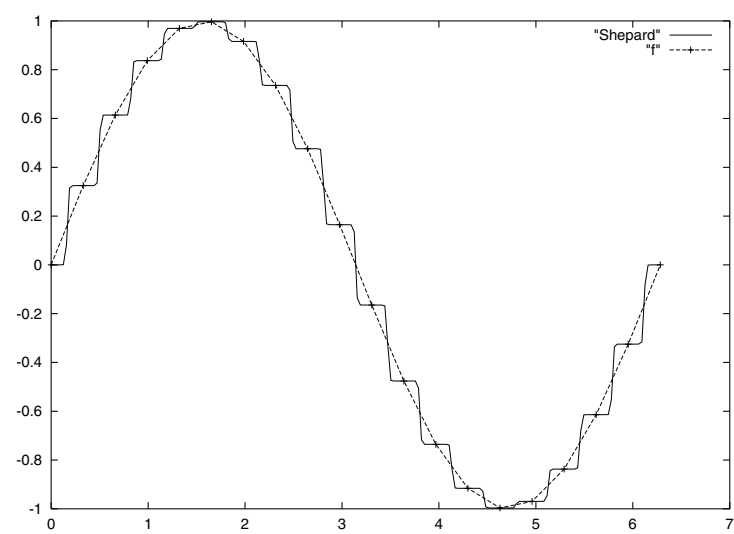

(d) $w^{(i)}(x)=\frac{1}{\left(x-x_{i}\right)^{12}}$

FiguRE 2. Shepard interpolants of $f(x)=\sin x$ with four different weight functions.

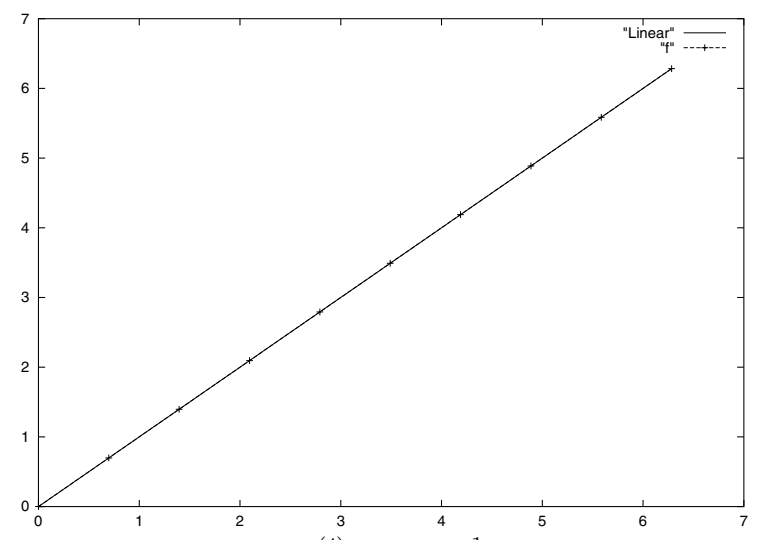

(a) $w^{(i)}(x)=\frac{1}{\left(x-x_{i}\right)^{2}}$

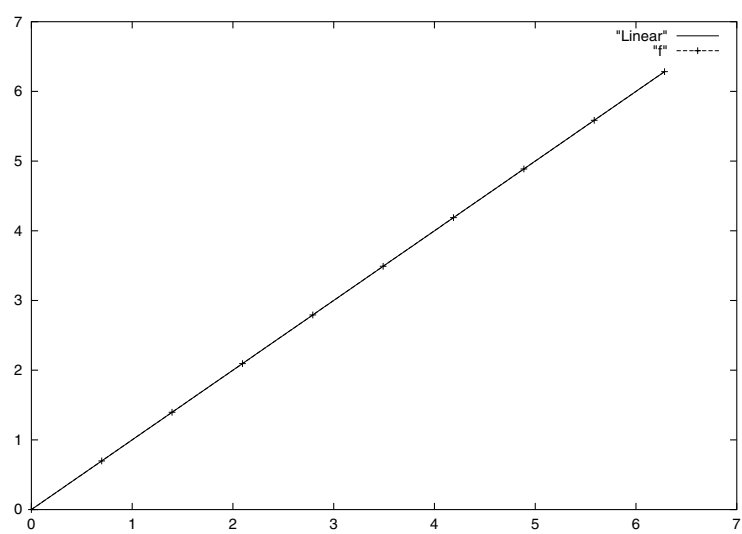

(b) $w^{(i)}(x)=\frac{{ }^{3}}{\left(x-x_{i}\right)^{12}}$

FiguRE 3. Interpolants of $f(x)=x$ with four different weight functions and a linear basis. 


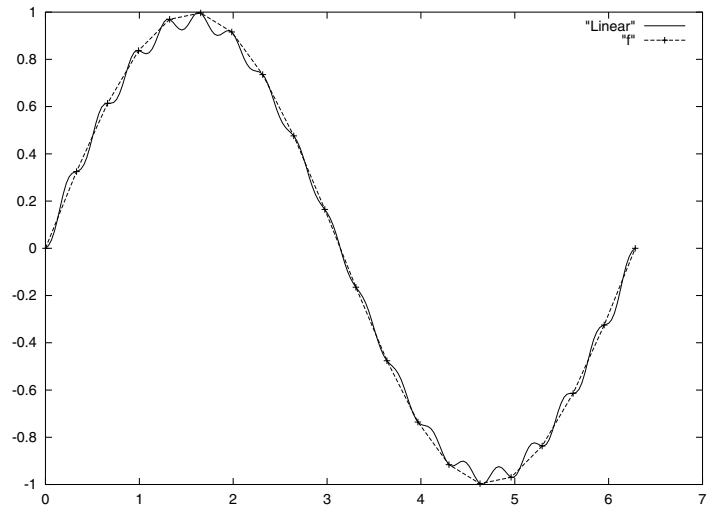

(a) $w^{(i)}(x)=\frac{1}{\left(x-x_{i}\right)^{2}}$

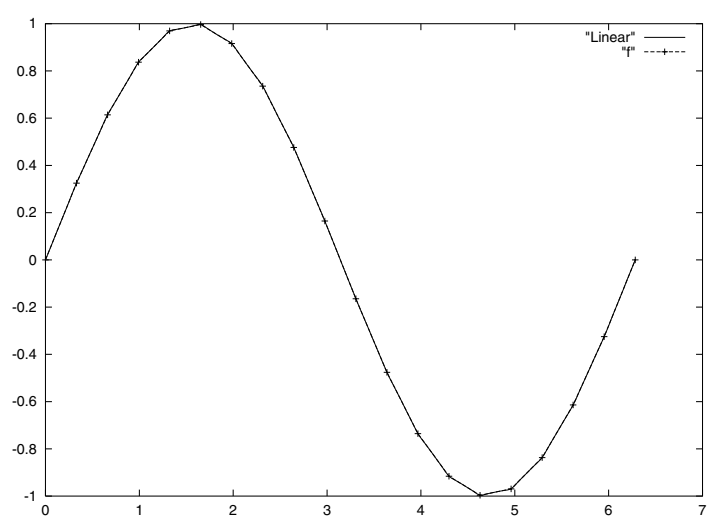

(c) $w^{(i)}(x)=\frac{1}{\left(x-x_{i}\right)^{8}}$

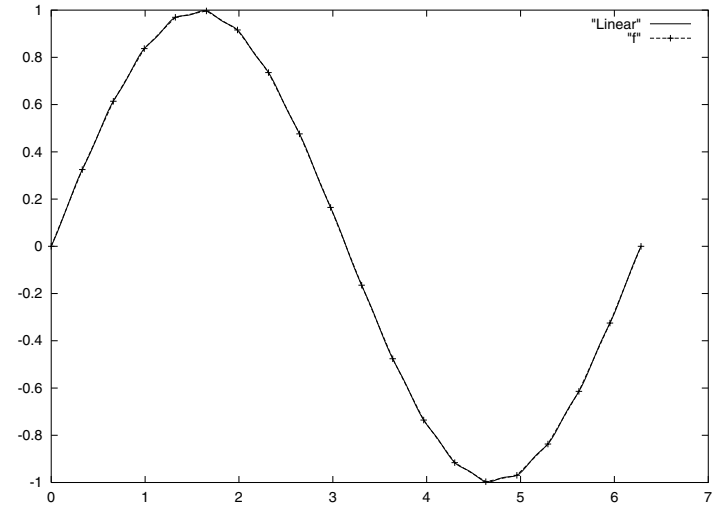

(b) $w^{(i)}(x)=\frac{1}{\left(x-x_{i}\right)^{4}}$

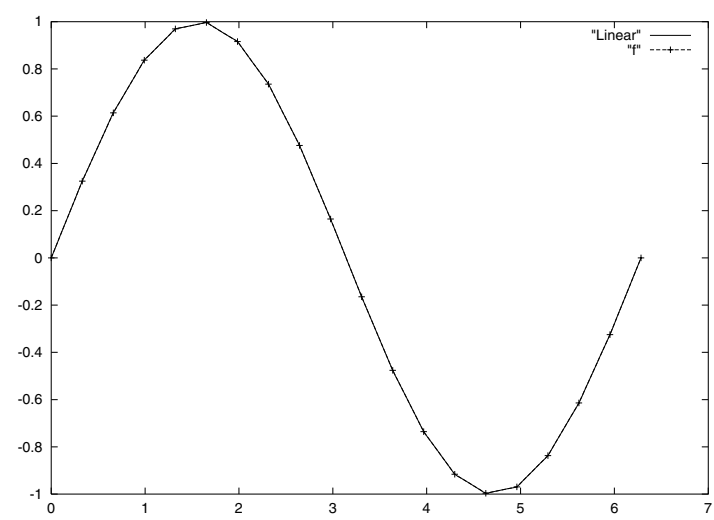

(d) $w^{(i)}(x)=\frac{1}{\left(x-x_{i}\right)^{12}}$

FIGURE 4. Interpolants of $f(x)=\sin x$ with four different weight functions in a linear polynomial basis.

\subsection{The case of the linear polynomial basis}

We discuss the case $n=2$, i.e. the case of the basis (2).

Remark 2.2 (note on notation). We have already introduced the notation $S f(x)$ for the Shepard interpolant. Here $S$ acts as an operator on $f$. If $f$ is simply the function $F(x)=x$ we will write $S x$ in order to be consistent with the general notation $S f(x)$. Consequently, $S x^{2}$ means $S f(x)$ where $f(x)=x^{2}$. The only case where we (have to) depart from this convention are functions of the form $f(x)=x+h$ where we write $S[x+h]$ in order to remind the reader that $S$ is an operator acting on the function $x \mapsto x+h$ and not a function of $x+h$.

According to Theorem 2.1 we compute

$$
\begin{aligned}
g^{(2)}(x) & =x-S x \\
\boldsymbol{U}(x) & =\left(x_{1}-S x, \ldots, x_{N}-S x\right) \\
\boldsymbol{U}(x) \boldsymbol{W}(x) \boldsymbol{B}^{\top}(x) & =\sum_{i=1}^{N} w^{(i)}(x) \cdot\left(x_{i}-S x\right)^{2} \\
\boldsymbol{U}(x) \boldsymbol{W}(x)\left(\boldsymbol{f}-S f(x) \boldsymbol{b}^{(1)}\right) & =\sum_{i=1}^{N} w^{(i)}(x) \cdot\left(x_{i}-S x\right) \cdot\left(f_{i}-S f(x)\right)
\end{aligned}
$$




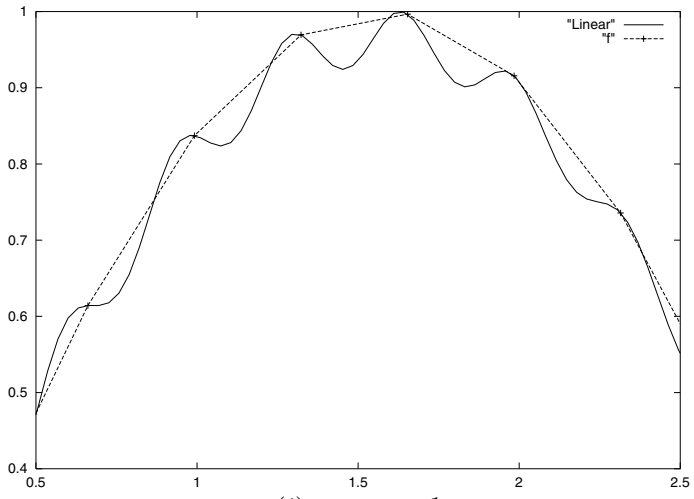

(a) $w^{(i)}(x)=\frac{1}{\left(x-x_{i}\right)^{2}}$

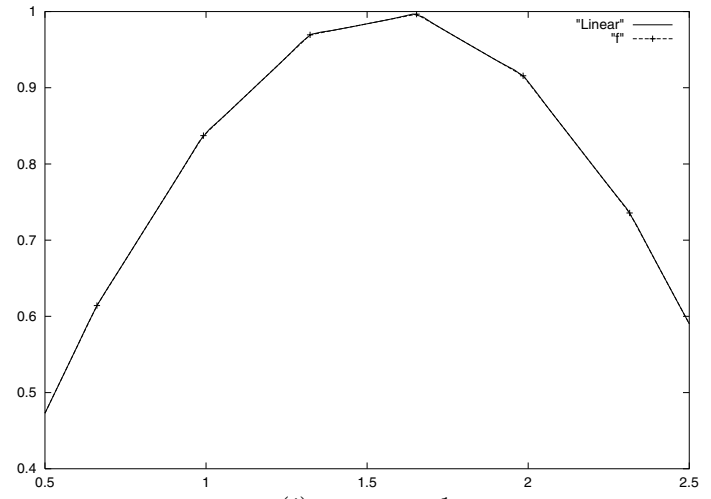

(b) $w^{(i)}(x)=\frac{1}{\left(x-x_{i}\right)^{12}}$

FIGURE 5. Details of the interpolants of $f(x)=\sin x$ showing the dimpling phenomenon under two different weight functions and a linear basis.

and hence we arrive at

$$
\beta_{2}(x)=\frac{\sum_{i=1}^{N} w^{(i)}(x) \cdot\left(x_{i}-S x\right) \cdot\left(f_{i}-S f(x)\right)}{\sum_{i=1}^{N} w^{(i)}(x) \cdot\left(x_{i}-S x\right)^{2}}, \quad x \notin\left\{x_{1}, \ldots, x_{N}\right\} .
$$

Since $g^{(2)}(x)=x-S x$ vanishes at every node $x_{k}$ the value of $\beta_{2}\left(x_{k}\right)$ is not of interest for the pure interpolation problem. However, this value will play an important role in computing derivatives and we will come back to it in the sequel.

In order to compare the numerical results we conclude this section with numerical experiments. We start again with interpolation of $f(x)=x$ with all parameters exactly as in the Shepard case.

As can be seen the basis (2) is perfect in interpolating a linear polynomial. In the case of $f(x)=\sin x$ with parameters as in the Shepard case we get the results shown in Figure 4.

In a closer look at the first local maximum of the sine function in Figure 5 the so called dimpling phenomenon can be seen which was already described by Lancaster and Šalkauskas in [7]. This is a clear sign that the linear basis is not well suited to interpolate quadratic-like shapes. However, as can also be seen from Figure 5 the dimpling phenomenon depends on the singularity of the weights used.

\subsection{The case of the quadratic polynomial basis}

We end our numerical excursion with the discussion of the case $n=3$, i.e. the case of the quadratic basis (3). According to Theorem 2.1 we compute

$$
\begin{aligned}
& g^{(2)}(x)=x-S x \\
& g^{(3)}(x)=x^{2}-S x^{2} \\
& \boldsymbol{U}(x)=\left(\begin{array}{ccc}
x_{1}-S x & \cdots & x_{N}-S x \\
x_{1}^{2}-S x^{2} & \cdots & x_{N}^{2}-S x^{2}
\end{array}\right) \\
& \boldsymbol{M}(x):=\boldsymbol{U}(x) \boldsymbol{W}(x) \boldsymbol{U}^{\top}(x) \\
& =\left(\begin{array}{lc}
\sum_{i=1}^{N} w^{(i)}(x) \cdot\left(x_{i}-S x\right)^{2} & \sum_{i=1}^{N} w^{(i)}(x) \cdot\left(x_{i}^{2}-S x^{2}\right) \cdot\left(x_{i}-S x\right) \\
\sum_{i=1}^{N} w^{(i)}(x) \cdot\left(x_{i}^{2}-S x^{2}\right) \cdot\left(x_{i}-S x\right) & \sum_{i=1}^{N} w^{(i)}(x) \cdot\left(x_{i}^{2}-S x^{2}\right)^{2}
\end{array}\right) \\
& \boldsymbol{U}(x) \boldsymbol{W}(x)\left(\boldsymbol{f}-S f(x) \boldsymbol{b}^{(1)}\right) \\
& =\left(\begin{array}{l}
\sum_{i=1}^{N} w^{(i)} \cdot\left(x_{i}-S x\right) \cdot\left(f_{i}-S f(x)\right) \\
\sum_{i=1}^{N} w^{(i)} \cdot\left(x_{i}^{2}-S x^{2}\right) \cdot\left(f_{i}-S f(x)\right)
\end{array}\right)
\end{aligned}
$$


and hence, for all $x \notin\left\{x_{1}, \ldots, x_{N}\right\}$,

$$
\begin{aligned}
\beta_{2}(x)= & \frac{1}{\Delta}\left\{\left(\sum_{i=1}^{N} w^{(i)}(x) \cdot\left(x_{i}^{2}-S x^{2}\right)^{2}\right) \cdot\left(\sum_{i=1}^{N} w^{(i)}(x) \cdot\left(x_{i}-S x\right) \cdot\left(f_{i}-S f(x)\right)\right)\right. \\
& \left.-\left(\sum_{i=1}^{N} w^{(i)}(x) \cdot\left(x_{i}^{2}-S x^{2}\right) \cdot\left(x_{i}-S x\right)\right) \cdot\left(\sum_{i=1}^{N} w^{(i)}(x) \cdot\left(x_{i}^{2}-S x^{2}\right) \cdot\left(f_{i}-S f(x)\right)\right)\right\} \\
\beta_{3}(x)= & \frac{1}{\Delta}\left\{\left(\sum_{i=1}^{N} w^{(i)}(x) \cdot\left(x_{i}-S x\right)^{2}\right) \cdot\left(\sum_{i=1}^{N} w^{(i)}(x) \cdot\left(x_{i}^{2}-S x^{2}\right) \cdot\left(f_{i}-S f(x)\right)\right)\right. \\
& \left.-\left(\sum_{i=1}^{N} w^{(i)}(x) \cdot\left(x_{i}^{2}-S x^{2}\right) \cdot\left(x_{i}-S x\right)\right) \cdot\left(\sum_{i=1}^{N} w^{(i)}(x) \cdot\left(x_{i}-S x\right) \cdot\left(f_{i}-S f(x)\right)\right)\right\},
\end{aligned}
$$

where

$$
\Delta:=\operatorname{det} M(x)
$$

As was the case with the linear basis the node values of $\beta_{2}, \beta_{3}$ do not play a role in the pure interpolation problem, since $g^{(2)}$ and $g^{(3)}$ vanish at the nodes.

We conclude again with the numerical experiments. Numerical tests with $f(x)=\sin x$ are $\operatorname{shown}^{1}$ in Figure 6 . The case of an inverse quadratic weight still exhibits a dimpling phenomenon which is completely gone already with an inverse quartic weight.

\section{Finite DifFERENCE OPERATORS FROM PSEUdOSPECTRAL INTERPOLANTS}

In pseudospectral methods for partial differential equations (see e.g. [2]) global interpolants are used on a given grid for purposes of approximation. Without diving too deep into the theory of these approximation techniques we follow Fornberg [4] in his interpretation of pseudospectral methods as global finite difference methods. On a grid with $N$ points $x_{1}, \ldots, x_{N}$ a polynomial of degree at most $N-1$ can be uniquely described. Fornberg solves the task:

Find coefficients $c_{i, j}^{k}$ such that the approximations

$$
\sum_{j=1}^{i} c_{i, j}^{k} f\left(x_{j}\right) \approx \frac{\mathrm{d}^{k} f}{\mathrm{~d} x^{k}}(x), \quad k=0,1, \ldots, m, \quad i=k, k+1, \ldots, N
$$

are optimal, i.e. exact for polynomials of degree as high as possible by means of a simple algorithm. See [3] for a proof that the given algorithms in fact produce the required optimal coefficients. We are interested in the first and second derivative, respectively, i.e. the cases $k=1$ and $k=2$. On an equidistant grid with three points $x_{1}, x_{2}, x_{3}$ and $h:=x_{2}-x_{1}$ the optimal central difference formulae are given by

$$
\begin{aligned}
D_{1} f(x) & :=c_{2,1}^{1} f\left(x_{1}\right)+c_{2,2}^{1} f\left(x_{2}\right)+c_{2,3}^{1} f\left(x_{3}\right) \\
& =-\frac{1}{2 h} f\left(x_{1}\right)+\frac{1}{2 h} f\left(x_{3}\right)=\frac{f\left(x_{3}\right)-f\left(x_{1}\right)}{2 h} \\
D_{2} f(x) & :=c_{3,1}^{2} f\left(x_{1}\right)+c_{3,2}^{2} f\left(x_{2}\right)+c_{3,3}^{2} f\left(x_{3}\right) \\
& =\frac{1}{h^{2}} f\left(x_{1}\right)-\frac{2}{h^{2}} f\left(x_{2}\right)+\frac{1}{h^{2}} f\left(x_{3}\right)=\frac{f\left(x_{1}\right)-2 f\left(x_{2}\right)+f\left(x_{3}\right)}{h^{2}} .
\end{aligned}
$$

\footnotetext{
1 The test case $f(x)=x$ is of course treated perfectly by the quadratic basis. In this case $\beta_{2}(x) \equiv 1$ so that the interpolant is the basis function $b^{(2)}(x)=x$.
} 


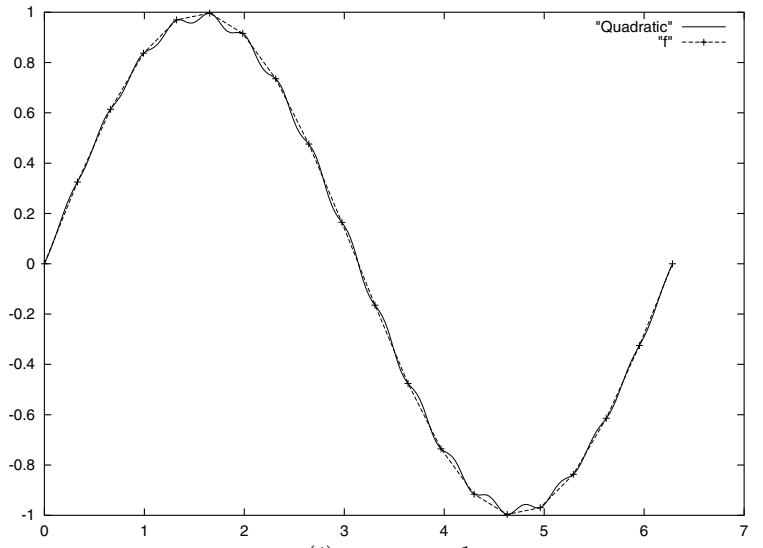

(a) $w^{(i)}(x)=\frac{1}{\left(x-x_{i}\right)^{2}}$

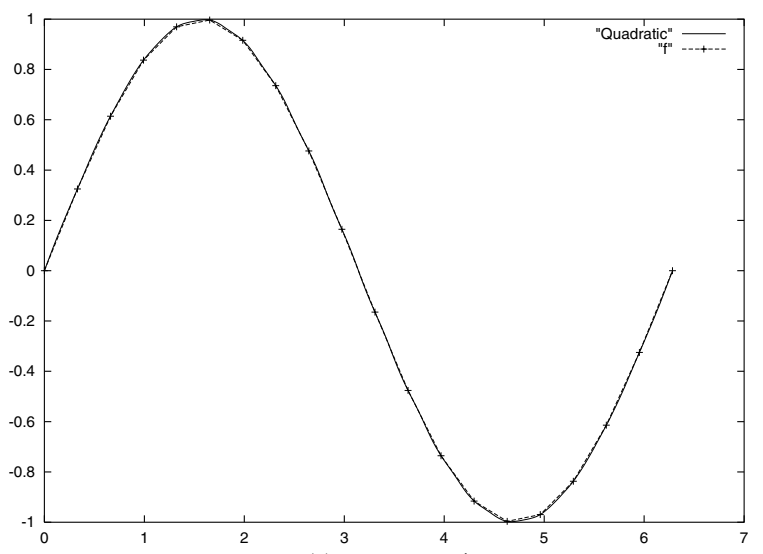

(c) $w^{(i)}(x)=\frac{1}{\left(x-x_{i}\right)^{4}}$

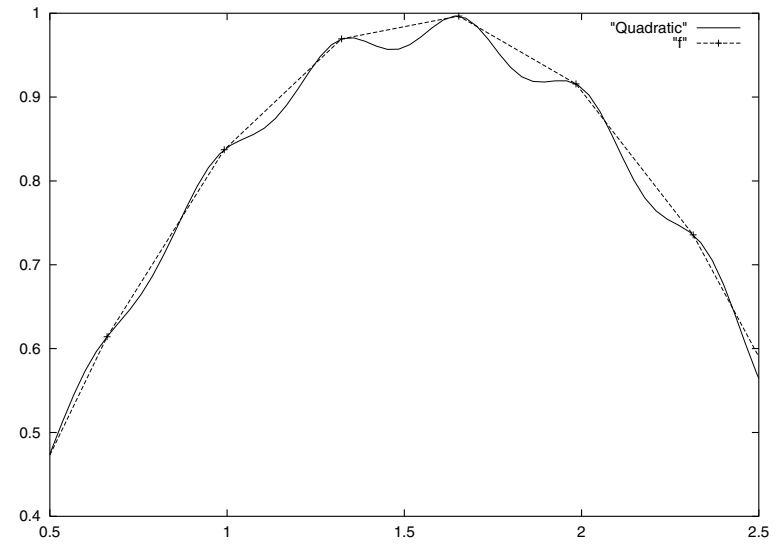

(b) $w^{(i)}(x)=\frac{1}{\left(x-x_{i}\right)^{2}}$

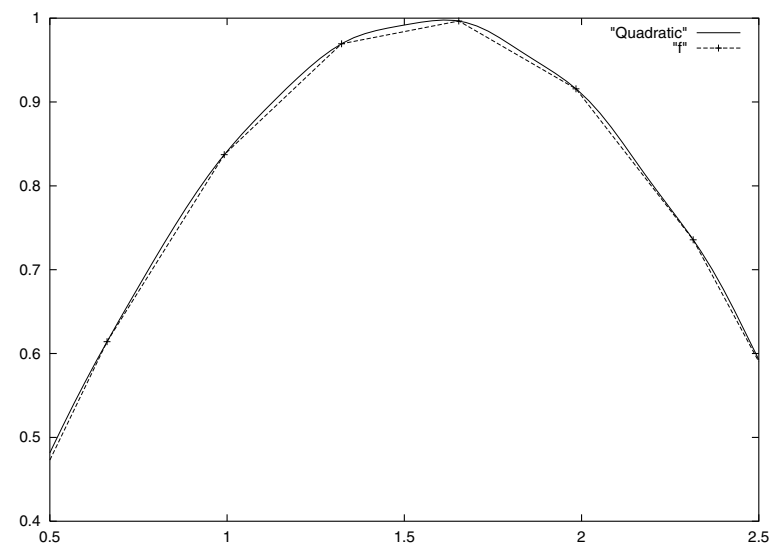

(d) $w^{(i)}(x)=\frac{1}{\left(x-x_{i}\right)^{4}}$

FIGURE 6. Interpolants of $f(x)=\sin x$ with two different weight functions in a quadratic polynomial basis.

For the case $h=1$ tables can be found in [4] giving the coefficients corresponding to the order.

If we reorder the nodes around a central node $x_{0}$ so that $x_{-1}, x_{1}$ are the neighbours to the left and right of $x_{0}$, respectively, and so on, Fornberg was able to compute growth rates of the optimal order coefficients. If $p$ denotes the even order of accuracy then the optimal coefficients for the first derivative satisfy

$$
c_{p, j}^{1}=\left\{\begin{array}{cl}
\frac{(-1)^{j+1}\left(\frac{p}{2} !\right)^{2}}{j\left(\frac{p}{2}+j\right) !\left(\frac{p}{2}-j\right) !} & ; j= \pm 1, \pm 2, \ldots, \pm \frac{p}{2} \\
0 \quad & ; j=0 .
\end{array}\right.
$$

In the limit $p \rightarrow \infty$ the growth rate of the coefficients is thus given by

$$
c_{\infty, j}^{1}=\left\{\begin{array}{cl}
\frac{(-1)^{j+1}}{j} ; & j= \pm 1, \pm 2, \ldots \\
0 ; & j=0 .
\end{array}\right.
$$


The corresponding growth rates for the second derivatives are given by

$$
c_{p, j}^{2}=\left\{\begin{aligned}
\frac{2 c_{p, j}^{1}}{j} ; & j= \pm 1, \pm 2, \ldots, \pm \frac{p}{2} \\
-2 \sum_{i=1}^{p / 2} \frac{1}{i^{2}} ; j & =0
\end{aligned}\right.
$$

and

$$
c_{\infty, j}^{2}=\left\{\begin{array}{cl}
\frac{2(-1)^{j+1}}{j^{2}} ; & j= \pm 1, \pm 2, \ldots \frac{p}{2} \\
-\frac{\pi}{3} ; & j=0 .
\end{array}\right.
$$

We will now compute global difference formulae for the first and second derivatives from the IMSL interpolants and then start a comparison with the optimal coefficients.

\section{Derivatives of IMSL interpolants AT THE NODES}

As was seen in the previous sections the IMSL interpolant rests upon the Shepard interpolant which is then "augmented" in order to increase the approximation order. Since we are interested in the derivatives of the IMSL interpolant we need a basic result concerning the behaviour of the derivatives of the Shepard interpolant at the nodes.

Theorem 4.1. Let $\alpha \in \mathbf{N}$ be even and $w^{(i)}(x):=1 /\left(x-x_{i}\right)^{\alpha}$. Then the Shepard interpolant satisfies

$$
\frac{\mathrm{d}^{j}}{\mathrm{~d} x^{j}} S f\left(x_{k}\right)=0, \quad j=1,2, \ldots, \alpha-1
$$

at every node $x_{k}$.

Proof. Although the result for the first derivative is known and can be deduced from the properties of the weights $v^{(i)}$ alone we give a direct proof for structural reasons.

Since $S f(x)=\sum_{i=1}^{N} f_{i} v^{(i)}(x)$ and $v^{(i)}(x)=\frac{w^{(i)}(x)}{\sum_{\ell=1}^{N} w^{(\ell)}(x)}$ we get by the quotient rule

$$
\begin{aligned}
\frac{\mathrm{d}}{\mathrm{d} x} S f(x) & =\sum_{i=1}^{N} f_{i} \frac{\mathrm{d}}{\mathrm{d} x} v^{(i)}(x) \\
& =\sum_{i=1}^{N} f_{i} \underbrace{\frac{\frac{\mathrm{d} w^{(i)}}{\mathrm{d} x}(x)\left(\sum_{\ell=1}^{N} w^{(\ell)}(x)\right)-w^{(i)}(x)\left(\sum_{\ell=1}^{N} \frac{\mathrm{d} w^{(\ell)}}{\mathrm{d} x}(x)\right)}{\left(\sum_{\ell=1}^{N} w^{(\ell)}(x)\right)^{2}}}_{=: \frac{A}{B}} .
\end{aligned}
$$


Since $w^{(i)}(x)=1 /\left(x-x_{i}\right)^{\alpha}$ it follows $\frac{\mathrm{d} w{ }^{(i)}}{\mathrm{d} x}=\frac{-\alpha}{\left(x-x_{i}\right)^{\alpha+1}}$. Define $h$ by $x=x_{k}+h$ so that $x_{k}+h<x_{k+1}$. Hence,

$$
\begin{aligned}
A= & \frac{-\alpha}{\left(x_{k}-x_{i}+h\right)^{\alpha+1}}\left\{\frac{1}{\left(x_{k}-x_{1}+h\right)^{\alpha}}+\cdots+\frac{1}{\left(x_{k}-x_{k-1}+h\right)^{\alpha}}+\frac{1}{h^{\alpha}}\right. \\
& \left.+\frac{1}{\left(x_{k}-x_{k+1}+h\right)^{\alpha}}+\cdots+\frac{1}{\left(x_{k}-x_{N}+h\right)^{\alpha}}\right\}-\frac{1}{\left(x_{k}-x_{i}+h\right)^{\alpha}} \\
& \times\left\{\frac{-\alpha}{\left(x_{k}-x_{1}+h\right)^{\alpha+1}}+\cdots+\frac{-\alpha}{\left(x_{k}-x_{k-1}+h\right)^{\alpha+1}}+\frac{-\alpha}{h^{\alpha+1}}\right. \\
& \left.+\frac{-\alpha}{\left(x_{k}-x_{k+1}+h\right)^{\alpha+1}}+\cdots+\frac{-\alpha}{\left(x_{k}-x_{N}+h\right)^{\alpha+1}}\right\},
\end{aligned}
$$

so that for $k \neq i$ we have

$$
\frac{\mathrm{d}}{\mathrm{d} x} v^{(i)}\left(x_{k}+h\right)=\frac{\mathcal{O}\left(\frac{1}{h^{\alpha+1}}\right)}{\mathcal{O}\left(\frac{1}{h^{2 \alpha}}\right)}=\mathcal{O}\left(h^{\alpha-1}\right) .
$$

For $k=i$ the term of order $\mathcal{O}\left(\frac{\alpha}{h^{2 \alpha+1}}\right)$ cancels so that in that case also

We now proceed with

$$
\frac{\mathrm{d}}{\mathrm{d} x} v^{(i)}\left(x_{i}+h\right)=\mathcal{O}\left(h^{\alpha-1}\right) \text {. }
$$

$$
\begin{aligned}
\frac{\mathrm{d}^{2}}{\mathrm{~d} x^{2}} S f(x) & =\sum_{i=1}^{N} f_{i} \frac{\mathrm{d}^{2}}{\mathrm{~d} x^{2}} v^{(i)}(x) \\
& =\sum_{i=1}^{N} f_{i} \frac{C \cdot D-E \cdot A}{\left(\sum_{\ell=1}^{N} w^{(\ell)}(x)\right)^{4}}
\end{aligned}
$$

with

$$
\begin{aligned}
C & :=\frac{\mathrm{d}^{2} w^{(i)}}{\mathrm{d} x^{2}}(x) \sum_{\ell=1}^{N} w^{(\ell)}(x)-w^{(i)}(x) \sum_{\ell=1}^{N} \frac{\mathrm{d}^{2} w^{(\ell)}}{\mathrm{d} x^{2}}(x) \\
D & :=\left(\sum_{\ell=1}^{N} w^{(\ell)}(x)\right)^{2} \\
E & :=2\left(\sum_{\ell=1}^{N} w^{(\ell)}(x)\right)\left(\sum_{\ell=1}^{N} \frac{\mathrm{d} w,^{(\ell)}}{\mathrm{d} x}(x)\right)
\end{aligned}
$$

and $A$ as defined above. Replacing $x$ by $x_{k}+h$ as before and since $\frac{\mathrm{d}^{2} w^{(i)}}{\mathrm{d} x^{2}}(x)=\frac{\alpha(\alpha+1)}{\left(x-x_{1}\right)^{\alpha+2}}$ we get

$$
\begin{aligned}
C \cdot D= & {\left[\frac{\alpha(\alpha+1)}{\left(x_{k}-x_{i}+h\right)^{\alpha+2}} \times\left\{\frac{1}{\left(x_{k}-x_{1}+h\right)^{\alpha}}+\cdots+\frac{1}{\left(x_{k}-x_{k-1}+h\right)^{\alpha}}+\frac{1}{h^{\alpha}}\right.\right.} \\
& \left.+\frac{1}{\left(x_{k}-x_{k+1}+h\right)^{\alpha}}+\cdots+\frac{1}{\left(x_{k}-x_{N}+h\right)^{\alpha}}\right\}-\frac{1}{\left(x_{k}-x_{i}+h\right)^{\alpha}} \\
& \times\left\{\frac{\alpha(\alpha+1)}{\left(x_{k}-x_{1}+h\right)^{\alpha+2}}+\cdots+\frac{\alpha(\alpha+1)}{\left(x_{k}-x_{k-1}+h\right)^{\alpha+2}}+\frac{\alpha(\alpha+1)}{h^{\alpha+2}}\right. \\
& \left.\left.+\frac{\alpha(\alpha+1)}{\left(x_{k}-x_{k+1}+h\right)^{\alpha+2}}+\cdots+\frac{\alpha(\alpha+1)}{\left(x_{k}-x_{N}+h\right)^{\alpha+2}}\right\}\right] \\
& \times\left(\frac{1}{\left(x_{k}-x_{1}+h\right)^{\alpha}}+\cdots+\frac{1}{h^{\alpha}}+\cdots \frac{1}{\left(x_{k}-x_{N}+h\right)^{\alpha}}\right)^{2} .
\end{aligned}
$$


Hence, $C \cdot D$ is of order $\mathcal{O}\left(\frac{1}{h^{3 \alpha+2}}\right)$ for $k \neq i$ while for $k=i$ the terms of order $\mathcal{O}\left(\frac{1}{h^{4 \alpha+2}}\right)$ cancel. Inspection of $E \cdot A$ also yields an order of $\mathcal{O}\left(\frac{1}{h^{3 \alpha+2}}\right)$. Together with the order $\mathcal{O}\left(\frac{1}{h^{4 \alpha}}\right)$ of the denominator we therefore arrive at

$$
\frac{\mathrm{d}^{2}}{\mathrm{~d} x^{2}} v^{(i)}(x)=\frac{\mathcal{O}\left(\frac{1}{h^{3 \alpha+2}}\right)}{\mathcal{O}\left(\frac{1}{h^{4 \alpha}}\right)}=\mathcal{O}\left(h^{\alpha-2}\right)
$$

Now we proceed by induction and note that the cancellation mechanism used twice is implied by the Leibniz rule for differentiating a product.

With the help of this useful Theorem we are now ready to compute the derivatives of the IMSL interpolants.

\subsection{Derivatives of the interpolant with linear basis}

We concentrate on the IMSL interpolant with linear basis

$$
G f(x)=S f(x)+\beta_{2}(x) g^{(2)}(x)=S f(x)+\beta_{2}(x)(x-S x)
$$

where $\beta_{2}$ is given for $x \notin\left\{x_{1}, \ldots, x_{N}\right\}$ by (8).

Lemma 4.1. The value of $\beta_{2}$ at a node $x_{k}$ exists and is given by

$$
\beta_{2}\left(x_{k}\right)=\frac{\sum_{\substack{i=1 \\ i \neq k}}^{n} w^{(i)}\left(x_{k}\right) \cdot\left(x_{i}-x_{k}\right)\left(f_{i}-f_{k}\right)}{\sum_{\substack{i=1 \\ i \neq k}}^{n} w^{(i)}\left(x_{k}\right) \cdot\left(x_{i}-x_{k}\right)^{2}} .
$$

Proof. Introducing $h$ by $x=x_{k}+h$ so that $x_{k}+h<x_{k+1}$ we consider

$$
\beta_{2}\left(x_{k}+h\right)=\frac{\sum_{i=1} w^{(i)}\left(x_{k}+h\right) \cdot\left(x_{i}-S\left[x_{k}+h\right]\right) \cdot\left(f_{i}-S f\left(x_{k}+h\right)\right)}{\sum_{i=1} w^{(i)}\left(x_{k}+h\right) \cdot\left(x_{i}-S\left[x_{k}+h\right]\right)^{2}} .
$$

The only terms which may become singular for $h \rightarrow 0$ are

$$
K(h):=w^{(k)}\left(x_{k}+h\right) \cdot\left(x_{k}-S\left[x_{k}+h\right]\right) \cdot\left(f_{k}-S f\left(x_{k}+h\right)\right)
$$

in the nominator and

$$
L(h):=w^{(k)}\left(x_{k}+h\right) \cdot\left(x_{k}-S\left[x_{k}+h\right]\right)^{2}
$$

in the denominator. Due to Theorem 4.1 a Taylor series gives

$$
\begin{aligned}
S\left[x_{k}+h\right] & =S x_{k}+h \frac{\mathrm{d}}{\mathrm{d} x} S x_{k}+\cdots+\frac{h^{\alpha-1}}{(\alpha-1) !} \frac{\mathrm{d}^{\alpha-1}}{\mathrm{~d} x^{\alpha-1}} S x_{k}+\mathcal{O}\left(h^{\alpha}\right) \\
& =x_{k}+\mathcal{O}\left(h^{\alpha}\right)
\end{aligned}
$$


and, analogously

$$
S f\left(x_{k}+h\right)=f_{k}+\mathcal{O}\left(h^{\alpha}\right) .
$$

Hence,

$$
K(h)=\mathcal{O}\left(h^{-\alpha}\right) \cdot \mathcal{O}\left(h^{\alpha}\right) \cdot \mathcal{O}\left(h^{\alpha}\right)=\mathcal{O}\left(h^{\alpha}\right)
$$

and

$$
L(h)=\mathcal{O}\left(h^{-\alpha}\right) \cdot \mathcal{O}\left(h^{2 \alpha}\right)=\mathcal{O}\left(h^{\alpha}\right) .
$$

Thus,

$$
\beta_{2}\left(x_{k}\right)=\lim _{h \rightarrow 0} \beta_{2}\left(x_{k}+h\right)=\frac{\sum_{\substack{i=1 \\ i \neq k}}^{n} w^{(i)}\left(x_{k}\right) \cdot\left(x_{i}-x_{k}\right)\left(f_{i}-f_{k}\right)}{\sum_{\substack{i=1 \\ i \neq k}}^{n} w^{(i)}\left(x_{k}\right) \cdot\left(x_{i}-x_{k}\right)^{2}}
$$

which is the required result.

Lemma 4.2. The value of $\frac{\mathrm{d}}{\mathrm{d} x} \beta_{2}$ at node $x_{k}$ exists and is given by

$$
\frac{\mathrm{d} \beta_{2}}{\mathrm{~d} x}\left(x_{k}\right)=\frac{A \cdot B-C \cdot D}{\left(\sum_{\substack{i=1 \\ i \neq k}}^{N} w^{(i)}\left(x_{k}\right)\left(x_{i}-x_{k}\right)^{2}\right)^{2}}
$$

with

$$
\begin{aligned}
A & :=\sum_{\substack{i=1 \\
i \neq k}}^{N} \frac{\mathrm{d} w^{(i)}}{\mathrm{d} x}\left(x_{k}\right)\left(x_{i}-x_{k}\right)\left(f_{i}-f_{k}\right) \\
B & :=\sum_{\substack{i=1 \\
i \neq k}}^{N} w^{(i)}\left(x_{k}\right)\left(x_{i}-x_{k}\right)^{2} \\
C & :=\sum_{\substack{i=1 \\
i \neq k}}^{N} w^{(i)}\left(x_{k}\right)\left(x_{i}-x_{k}\right)\left(f_{i}-f_{k}\right) \\
D & :=\sum_{\substack{i=1 \\
i \neq k}}^{N} \frac{\mathrm{d} w^{(i)}}{\mathrm{d} x}\left(x_{k}\right)\left(x_{i}-x_{k}\right)^{2} .
\end{aligned}
$$

Proof. Taking the derivative of $\beta_{2}$ for $x \notin\left\{x_{1}, \ldots, x_{N}\right\}$ yields

$$
\frac{\mathrm{d} \beta_{2}}{\mathrm{~d} x}(x)=\frac{(a-b-c) \cdot d-e \cdot f}{\left(\sum_{i=1}^{N} w^{(i)}(x) \cdot\left(x_{i}-S x\right)^{2}\right)^{2}}
$$


with

$$
\begin{aligned}
a & :=\sum_{i=1}^{N} \frac{\mathrm{d} w^{(i)}}{\mathrm{d} x}(x) \cdot\left(x_{i}-S x\right) \cdot\left(f_{i}-S f(x)\right) \\
b & :=\sum_{i=1}^{N} w^{(i)}(x) \cdot \frac{\mathrm{d} S x}{\mathrm{~d} x}(x) \cdot\left(f_{i}-S f(x)\right) \\
c & :=\sum_{i=1}^{N} w^{(i)}(x) \cdot\left(x_{i}-S x\right) \cdot \frac{\mathrm{d} S f}{\mathrm{~d} x}(x) \\
d & :=\sum_{i=1}^{N} w^{(i)}(x) \cdot\left(x_{i}-S x\right)^{2} \\
e & :=\sum_{i=1}^{N} w^{(i)}(x) \cdot\left(x_{i}-S x\right) \cdot\left(f_{i}-S f(x)\right) \\
f & :=\sum_{i=1}^{N} \frac{\mathrm{d} w^{(i)}}{\mathrm{d} x}(x) \cdot\left(x_{i}-S x\right)^{2}-2 w^{(i)}(x) \cdot\left(x_{i}-S x\right) \frac{\mathrm{d} S x}{\mathrm{~d} x}(x) .
\end{aligned}
$$

Introducing $x=x_{k}+h$ we see that singularities may occur only if $i=k$. In this case the product $(a-b-c) \cdot d$ gives an order of

$$
\begin{aligned}
O_{1}:= & \left(\mathcal{O}\left(\frac{1}{h^{\alpha+1}}\right) \cdot \mathcal{O}\left(h^{\alpha}\right) \cdot \mathcal{O}\left(h^{\alpha}\right) \cdot-\mathcal{O}\left(\frac{1}{h^{\alpha}}\right) \cdot \mathcal{O}\left(h^{\alpha-1}\right) \cdot \mathcal{O}\left(h^{\alpha}\right)\right. \\
& \left.-\mathcal{O}\left(\frac{1}{h^{\alpha}}\right) \cdot \mathcal{O}\left(h^{\alpha}\right) \cdot \mathcal{O}\left(h^{\alpha-1}\right)\right) \cdot\left(\mathcal{O}\left(\frac{1}{h^{\alpha}}\right) \cdot \mathcal{O}\left(h^{2 \alpha}\right)\right) \\
= & \mathcal{O}\left(h^{2 \alpha-1}\right) .
\end{aligned}
$$

Analogously, the product $e \cdot f$ results in

$$
\mathrm{O}_{2}:=\mathcal{O}\left(h^{2 \alpha-1}\right)
$$

while the case $i=k$ results in the denominator in a term of order

$$
O_{3}:=\mathcal{O}\left(h^{2 \alpha}\right)
$$

Hence, no singularity occurs for $h \rightarrow 0$. In that case $b, c$ and the summand in $f$ vanishes as the first derivative of the Shepard interpolant vanishes at the nodes. Furthermore, $S x_{k}=x_{k}$ and $S f\left(x_{k}\right)=f_{k}$ and the formula stated follows.

Lemma 4.3. The first derivative of the IMSL interpolant based on the linear basis (2) at the node $x_{k}$ is given by

$$
\frac{\mathrm{d} G f}{\mathrm{~d} x}\left(x_{k}\right)=\beta_{2}\left(x_{k}\right)
$$

If the exponent in the weights $w^{(i)}$ is $\alpha>2$ then the second derivative at the nodes is given by

$$
\frac{\mathrm{d}^{2} G f}{\mathrm{~d} x^{2}}\left(x_{k}\right)=2 \frac{\mathrm{d} \beta_{2}}{\mathrm{~d} x}\left(x_{k}\right)
$$


Proof. Since $G f(x)=S f(x)+\beta_{2}(x)(x-S x)$ we get

$$
\frac{\mathrm{d} G f}{\mathrm{~d} x}(x)=\frac{\mathrm{d} S f}{\mathrm{~d} x}(x)+\frac{\mathrm{d} \beta_{2}}{\mathrm{~d} x}(x)(x-S x)+\beta_{2}(x)\left(1-\frac{\mathrm{d} S x}{\mathrm{~d} x}(x)\right)
$$

and

$$
\begin{aligned}
\frac{\mathrm{d}^{2} G f}{\mathrm{~d} x^{2}}(x)= & \frac{\mathrm{d}^{2} S f}{\mathrm{~d} x^{2}}(x)+\frac{\mathrm{d}^{2} \beta_{2}}{\mathrm{~d} x^{2}}(x)(x-S x)+2 \frac{\mathrm{d} \beta_{2}}{\mathrm{~d} x}(x)\left(1-\frac{\mathrm{d} S x}{\mathrm{~d} x}(x)\right) \\
& -\beta_{2}(x) \frac{\mathrm{d}^{2} S x}{\mathrm{~d} x^{2}}(x) .
\end{aligned}
$$

The results now follow for $x=x_{k}$ since $S x_{k}=x_{k}$ and $\mathrm{d} S x / \mathrm{d} x\left(x_{k}\right)=0$. For the second derivative we exploit Theorem 4.1 to conclude $\mathrm{d}^{2} S x / \mathrm{d} x^{2}\left(x_{k}\right)=0$ under the given assumptions on $\alpha$.

Due to the flat spot phenomenon the Shepard interpolant will not result in a consistent first derivative. Here, consistency is meant in the finite difference sense, i.e. a derivative $D f$ is consistent, if and only if $D f \rightarrow \frac{\mathrm{d} f}{\mathrm{~d} x}$ for grid size $h \rightarrow 0$. It would therefore be strange if the IMLS interpolant based on the linear basis would give a consistent second derivative. In fact we can prove

Theorem 4.2. The second derivative $\frac{\mathrm{d}^{2} G f}{\mathrm{~d} x^{2}}$ of the IMLS interpolant based on the linear basis is inconsistent.

Proof. We consider $N=3$ since a consistent finite difference representation is possible with three grid points and evaluate at the point $x_{2}$. For simplicity renumber $x_{1}:=-1, x_{2}:=0, x_{3}=1$ by $x_{-1}, x_{0}, x_{1}$ and evaluate $\frac{\mathrm{d} \beta_{2}}{\mathrm{~d} x}$ at $x_{2}$, see Lemma 4.2. Then

$$
\frac{\mathrm{d} \beta_{2}}{\mathrm{~d} x}(0)=\frac{A \cdot B-C \cdot D}{\left(w^{(-1)}(0)+w^{(1)}(0)\right)^{2}}
$$

with

$$
\begin{aligned}
A & =\frac{\mathrm{d} w^{(-1)}}{\mathrm{d} x}(0)\left(f_{0}-f_{-1}\right)+\frac{\mathrm{d} w^{(1)}}{\mathrm{d} x}(0)\left(f_{1}-f_{0}\right) \\
B & =w^{(-1)}(0)+w^{(1)}(0) \\
C & =w^{(-1)}(0)\left(f_{0}-f_{-1}\right)+w^{(1)}(0)\left(f_{1}-f_{0}\right) \\
D & =\frac{\mathrm{d} w^{(-1)}}{\mathrm{d} x}(0)+\frac{\mathrm{d} w^{(1)}}{\mathrm{d} x}(0)
\end{aligned}
$$

Now $w^{(-1)}(0)=w^{(1)}(0)$ and $\frac{\mathrm{d} w^{(-1)}}{\mathrm{d} x}(0)=-\frac{\mathrm{d} w^{(1)}}{\mathrm{d} x}(0)$ so that $A=\frac{\mathrm{d} w^{(1)}}{\mathrm{d} x}(0)\left(f_{1}-2 f_{0}+f_{-1}\right), B=2 w^{(1)}(0)$, $C=w^{(1)}(0)\left(f_{1}-f_{-1}\right)$ and $D=0$. Hence,

$$
\frac{\mathrm{d} \beta_{2}}{\mathrm{~d} x}(0)=\frac{\frac{\mathrm{d} w^{(1)}}{\mathrm{d} x}(0)\left(f_{1}-2 f_{0}+f_{-1}\right) \cdot 2 w^{(1)}(0)}{\left(2 w^{(1)}(0)\right)^{2}}=\frac{\frac{\mathrm{d} w^{(1)}}{\mathrm{d} x}(0)\left(f_{1}-2 f_{0}+f_{-1}\right)}{2 w^{(1)}(0)} .
$$

Since $w^{(1)}(x)=1 /(x-1)^{\alpha}$ we have $w^{(1)}(0)=1$ and $\frac{\mathrm{d} w^{(1)}}{\mathrm{d} x}(0)=-\alpha /(-1)^{\alpha+1}=\alpha$ we end up with

$$
\frac{\mathrm{d} \beta_{2}}{\mathrm{~d} x}(0)=\frac{\alpha\left(f_{1}-2 f_{0}+f_{-1}\right)}{2} .
$$

Following Lemma 4.3 we see that

$$
\frac{\mathrm{d}^{2} G f}{\mathrm{~d} x}(0)=2 \frac{\mathrm{d} \beta_{2}}{\mathrm{~d} x}(0)=\alpha\left(f_{1}-2 f_{0}+f_{-1}\right)
$$




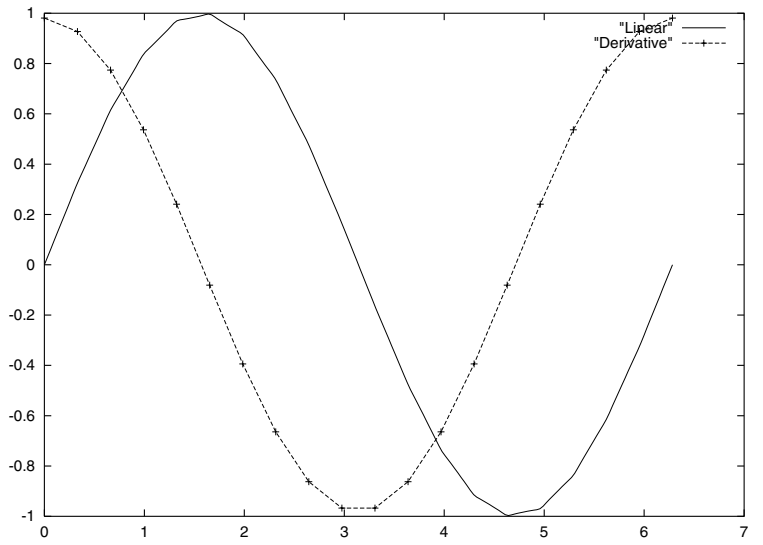

(a) $w^{(i)}(x)=\frac{1}{\left(x-x_{i}\right)^{8}}$

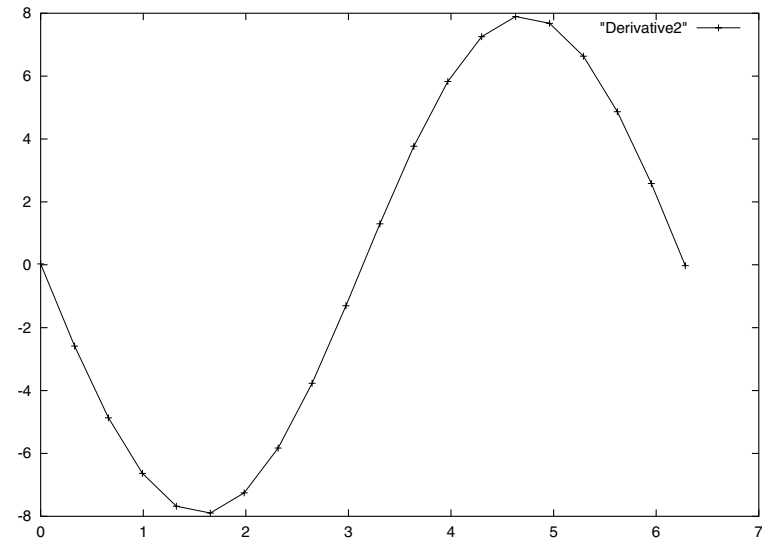

(b) $w^{(i)}(x)=\frac{1}{\left(x-x_{i}\right)^{8}}$

FIgURE 7. IMLS interpolant and first and second derivative of $f(x)=\sin x$ with weight function $1 /\left(x-x_{i}\right)^{8}$.

and since $f_{1}-2 f_{0}+f_{-1} \stackrel{.}{=} \frac{\mathrm{d}^{2} f}{\mathrm{~d} x^{2}}$ up to terms of second order the inconsistency manifests itself in a constant factor $\alpha>1$.

We conclude by presenting numerical experiments and use again 20 equispaced nodes for the sine function. In the left subfigure of Figure 7 the IMLS interpolant is shown on 200 plot points together with the first derivative on the 20 node points. In the right subfigure we see the second derivative showing clearly the kind of inconsistency predicted in Theorem 4.2. While the shape of the second derivative $(-\sin x)$ is clearly seen the constant factor $\alpha=8$ spoils the result.

Even more pronounced the problems with the second derivative can be seen with the function $x \mapsto x^{3}$ on $[0,2 \pi]$. Figure 8 shows the inconsistency as predicted by Theorem 4.2 but also a strange phenomenon at the boundaries. This is easily explainable from a finite difference viewpoint. At the boundaries the formulae for the derivatives correspond to one-sided differences which always are worse than centered differences concerning the consistency. It is therefore not astonishing that we see massive problems at the boundaries in our numerical tests.

\subsection{Derivatives of the interpolant with quadratic basis}

We now discuss the case of the basis (3), i.e.

$$
G f(x)=S f(x)+\beta_{2}(x)(x-S x)+\beta_{3}(x)\left(x^{2}-S x^{2}\right)
$$

where $\beta_{2}$ and $\beta_{3}$ are given by (9) and (10), respectively.

Lemma 4.4. The values of $\beta_{2}\left(x_{k}\right)$ and $\beta_{3}\left(x_{k}\right)$ exist and are given by

$$
\begin{aligned}
& \beta_{2}\left(x_{k}\right)=\frac{a_{22} \cdot r_{1}-a_{12} \cdot r_{2}}{\Delta}, \\
& \beta_{3}\left(x_{k}\right)=\frac{a_{11} \cdot r_{2}-a_{12} \cdot r_{1}}{\Delta},
\end{aligned}
$$




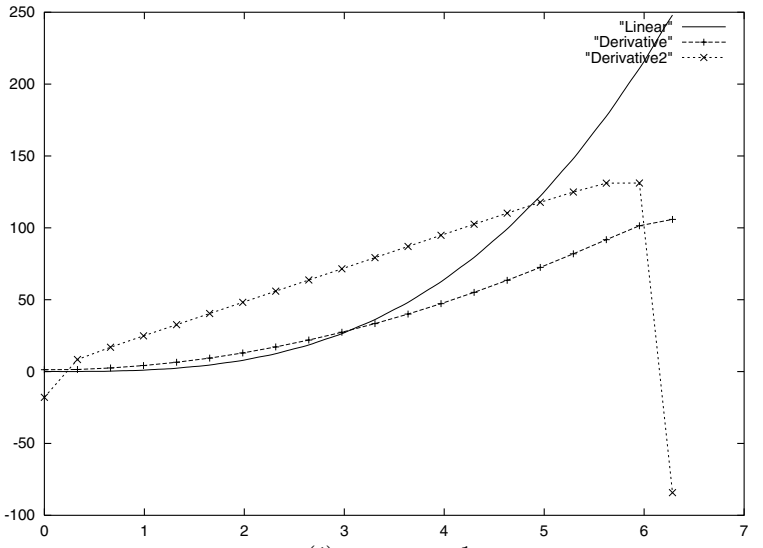

(a) $w^{(i)}(x)=\frac{1}{\left(x-x_{i}\right)^{4}}$

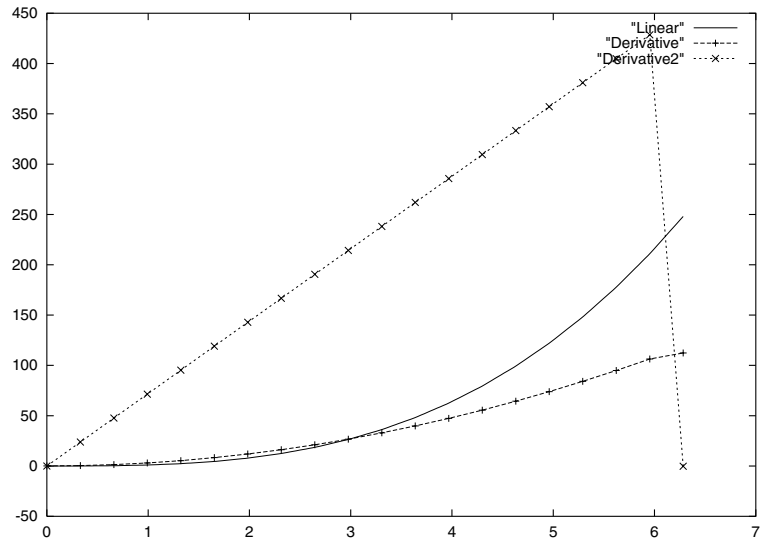

(b) $w^{(i)}(x)=\frac{1}{\left(x-x_{i}\right)^{12}}$

FIGURE 8. IMLS interpolant and first and second derivative of $f(x)=x^{3}$ with different weight functions showing massive problems with the second derivative at the boundaries.

where

$$
\begin{aligned}
a_{11} & :=\sum_{\substack{i=1 \\
i \neq k}}^{N} w^{(i)}\left(x_{k}\right) \cdot\left(x_{i}-x_{k}\right)^{2} \\
a_{12} & :=\sum_{\substack{i=1 \\
i \neq k}}^{N} w^{(i)}\left(x_{k}\right) \cdot\left(x_{i}^{2}-x_{k}^{2}\right) \cdot\left(x_{i}-x_{k}\right) \\
a_{22} & :=\sum_{\substack{i=1 \\
i \neq k}}^{N} w^{(i)}\left(x_{k}\right) \cdot\left(x_{i}^{2}-x_{k}^{2}\right)^{2} \\
r_{1} & :=\sum_{\substack{i=1 \\
i \neq k}}^{N} w^{(i)}\left(x_{k}\right) \cdot\left(x_{i}-x_{k}\right) \cdot\left(f_{i}-f_{k}\right) \\
r_{2} & :=\sum_{\substack{i=1 \\
i \neq k}}^{N} w^{(i)}\left(x_{k}\right) \cdot\left(x_{i}^{2}-x_{k}^{2}\right) \cdot\left(f_{i}-f_{k}\right) \\
\Delta & :=a_{11} \cdot a_{22}-a_{12}^{2} .
\end{aligned}
$$

Proof. With the notations above the functions $\beta_{2}$ and $\beta_{3}$ are defined at points $x \notin\left\{x_{1}, \ldots, x_{N}\right\}$ by (compare with (9), (10)),

$$
\begin{aligned}
& \beta_{2}\left(x_{k}\right)=\frac{\tilde{a}_{22} \cdot \tilde{r}_{1}-\tilde{a}_{12} \cdot \tilde{r}_{2}}{\tilde{\Delta}}, \\
& \beta_{3}\left(x_{k}\right)=\frac{\tilde{a}_{11} \cdot \tilde{r}_{2}-\tilde{a}_{12} \cdot \tilde{r}_{1}}{\tilde{\Delta}},
\end{aligned}
$$


where the ${ }^{\sim}$-terms are exactly the terms above but with $i=k$ allowed in the summation. An asymptotic analysis of the kind already carried out previously shows that in case of $x \rightarrow x_{k}$ the singular weights die out by multiplication with terms of positive order.

Lemma 4.5. The values of $\frac{\mathrm{d} \beta_{2}}{\mathrm{~d} x}\left(x_{k}\right)$ and $\frac{\mathrm{d} \beta_{3}}{\mathrm{~d} x}\left(x_{k}\right)$ exist and are given by

$$
\begin{aligned}
\frac{\mathrm{d} \beta_{2}}{\mathrm{~d} x}\left(x_{k}\right)= & \frac{1}{\Delta^{2}}\left\{\left(A_{22} \cdot r_{1}+a_{22} \cdot R_{1}-A_{12} \cdot r_{2}-a_{12} \cdot R_{2}\right) \cdot \Delta\right. \\
& \left.-\left(a_{22} \cdot r_{1}-a_{12} \cdot r_{2}\right) \cdot\left(A_{11} \cdot a_{22}+a_{11} \cdot A_{22}-2 a_{12} \cdot A_{12}\right)\right\} \\
\frac{\mathrm{d} \beta_{3}}{\mathrm{~d} x}\left(x_{k}\right)= & \frac{1}{\Delta^{2}}\left\{\left(A_{11} \cdot r_{2}+a_{11} \cdot R_{2}-A_{12} \cdot r_{1}-a_{12} \cdot R_{1}\right) \cdot \Delta\right. \\
& \left.-\left(a_{11} \cdot r_{2}-a_{12} \cdot r_{1}\right) \cdot\left(A_{11} \cdot a_{22}+a_{11} \cdot A_{22}-2 a_{12} \cdot A_{12}\right)\right\},
\end{aligned}
$$

where $a_{i j}, r_{i}$ are defined as in Lemma 4.4 and

$$
\begin{aligned}
A_{11}:= & \sum_{\substack{i=1 \\
i \neq k}}^{N} \frac{\mathrm{d} w^{(i)}}{\mathrm{d} x}\left(x_{k}\right) \cdot\left(x_{i}-x_{k}\right)^{2} \\
A_{12}:= & \sum_{\substack{i=1 \\
i \neq k}}^{N} \frac{\mathrm{d} w^{(i)}}{\mathrm{d} x}\left(x_{k}\right) \cdot\left(x_{i}^{2}-x_{k}^{2}\right) \cdot\left(x_{i}-x_{k}\right) \\
A_{22}:= & \sum_{\substack{i=1 \\
i \neq k}}^{N} \frac{\mathrm{d} w^{(i)}}{\mathrm{d} x}\left(x_{k}\right) \cdot\left(x_{i}^{2}-x_{k}^{2}\right)^{2} \\
R_{1}:= & \sum_{\substack{i=1 \\
i \neq k}}^{N} \frac{\mathrm{d} w^{(i)}}{\mathrm{d} x}\left(x_{k}\right) \cdot\left(x_{i}-x_{k}\right) \cdot\left(f_{i}-f_{k}\right) \\
R_{2}:= & \sum_{\substack{i=1 \\
i \neq k}}^{N} \frac{\mathrm{d} w^{(i)}}{\mathrm{d} x}\left(x_{k}\right) \cdot\left(x_{i}^{2}-x_{k}^{2}\right) \cdot\left(f_{i}-f_{k}\right) .
\end{aligned}
$$

Proof. Take derivatives of (9) and (10) and consider again the case $x \rightarrow x_{k}$ where an asymptotic analysis shows again that the singularity of $w^{(i)}$ and $\mathrm{d} w^{(i)} / \mathrm{d} x$ at $x_{k}$ die out by multiplication with terms of positive order.

Lemma 4.6. The first and second derivative of $G f$ at the nodes $x_{k}$ are given by

$$
\begin{aligned}
\frac{\mathrm{d} G f}{\mathrm{~d} x}\left(x_{k}\right) & =\beta_{2}\left(x_{k}\right)+2 x_{k} \beta_{3}\left(x_{k}\right) \\
\frac{\mathrm{d}^{2} G f}{\mathrm{~d} x^{2}}\left(x_{k}\right) & =2\left(\frac{\mathrm{d} \beta_{2}}{\mathrm{~d} x}\left(x_{k}\right)+\beta_{3}\left(x_{k}\right)\right)+4 x_{k} \frac{\mathrm{d} \beta_{3}}{\mathrm{~d} x}\left(x_{k}\right) .
\end{aligned}
$$

Proof. Proceed exactly as in the proof of Lemma 4.3.

As in the case of the IMLS interpolant based on the linear polynomial basis we now present results for the case of the quadratic basis. In the case of interpolation of $x \mapsto \sin x$ on $[0,2 \pi]$ we get the following, very interesting numerical results. In Figure 9a we see the IMLS interpolant, its first and second derivative on 20 nodes with weight exponent $\alpha=8$. The shape of the second derivative $(-\sin x)$ is clearly seen but there seem to be problems at the boundaries. In case these problems were consistency problems as in the case of the linear basis they would not decrease if $N$ would be increased. However, in Figure $9 \mathrm{~b}$ all parameters are unchanged but the number of nodes is increased to $N=200$. We see that the problems at the boundaries diminished 


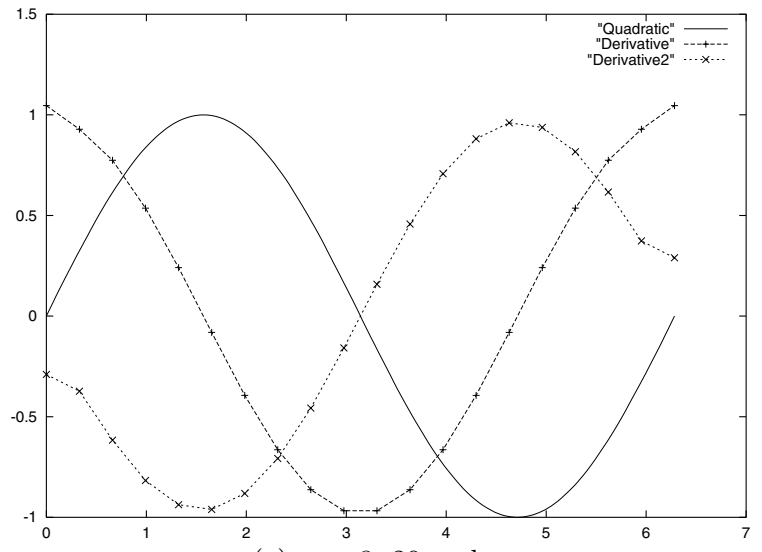

(a) $\alpha=8,20$ nodes

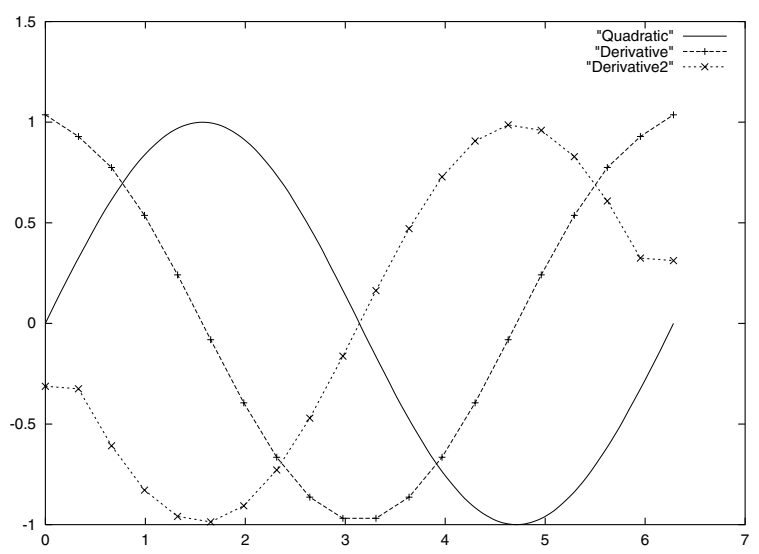

(c) $\alpha=12,20$ nodes

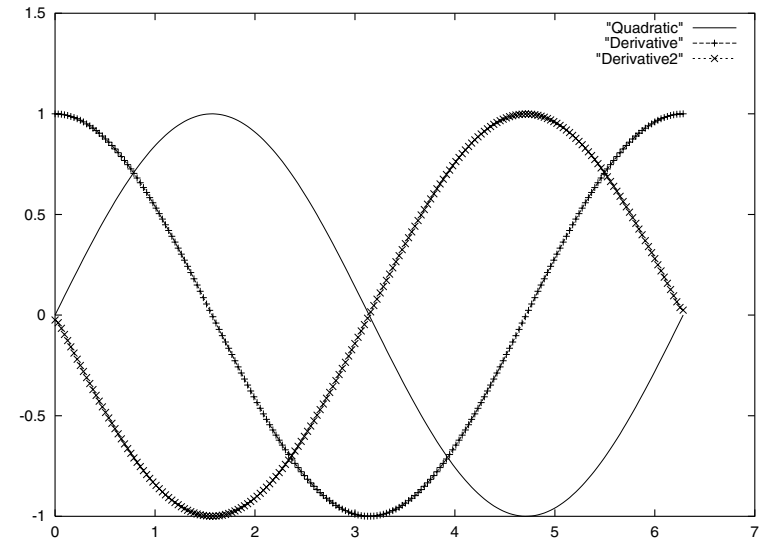

(b) $\alpha=8,100$ nodes

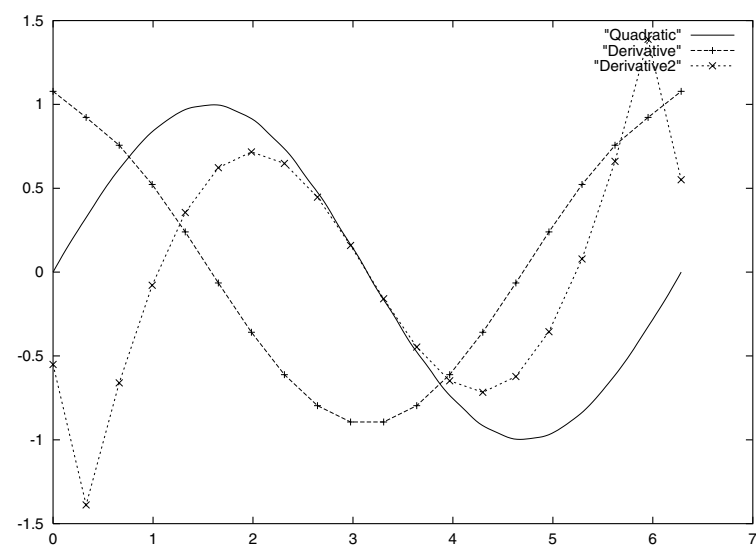

(d) $\alpha=4,20$ nodes

Figure 9. Interpolants of $f(x)=\sin x$ with first and second derivative using different weight functions and different number of nodes in a quadratic polynomial basis.

drastically. Which rôle is played by the weight function? In Figure 9c the number of nodes is again 20 but the weight exponent is $\alpha=12$. Obviously, the boundary problem is more pronounced as in the case of $\alpha=8$. Finally, in Figure 9d the weight exponent $\alpha=4$ is used which is a valid exponent. In that case consistency is lost completely and a useless second derivative appears. Numerical experiments show that $\alpha \geq 6$ leads to a consistent second derivative. In order to understand this behaviour we now start to examine the derivatives in terms of finite differences.

\section{A FINITE DIFFERENCE ANALYSIS OF IMLS DERIVATIVES}

\subsection{First derivatives}

We start by inspecting the first derivative

$$
\frac{\mathrm{d} G f}{\mathrm{~d} x}\left(x_{k}\right)=\beta_{2}(k)
$$


of the IMLS interpolant based on the linear polynomial basis where $\beta_{2}\left(x_{k}\right)$ was derived in Lemma 4.3. In order to compare with the coefficients of optimal difference formulae (11), (12) we change the enumeration to

$$
\ldots, x_{-2}:=x_{k-2}, x_{-1}:=x_{-1}, x_{0}:=x_{k}, x_{1}:=x_{k+1}, x_{2}:=x_{k+2}, \ldots
$$

define $h$ to be the mesh width and concentrate on central difference formulae. If the IMLS interpolant is constructed on a grid with $N$ points then the resulting difference formula will also include $N$ points. Thus, for the sake of comparision, we will consider the IMLS interpolant on exactly three points and will speak then of a "three-point grid". Note that this is just a technical trick to reduce the support of the interpolant. We could have restricted the support also by restricting the support of the weights which is very common in practice.

By simply evaluating $\beta_{2}$ at $x_{0}$ we get

Lemma 5.1. In case of a three-point grid the first derivative of the "linear" IMLS interpolant gives the finite difference formula

$$
\frac{\mathrm{d} G f}{\mathrm{~d} x}\left(x_{0}\right)=\frac{f_{1}-f_{-1}}{2 h}
$$

while the difference formula for a five-point grid is

$$
\frac{\mathrm{d} G f}{\mathrm{~d} x}\left(x_{0}\right)=\frac{f_{1}-f_{-1}}{2 h\left(1+\frac{4}{2^{\alpha}}\right)}+\frac{f_{2}-f_{-2}}{2^{\alpha} h\left(1+\frac{4}{2^{\alpha}}\right)} .
$$

On a seven-point grid the formula is given by

$$
\begin{aligned}
\frac{\mathrm{d} G f}{\mathrm{~d} x}\left(x_{0}\right)= & \frac{3^{\alpha}\left(f_{1}-f_{-1}\right)}{h\left(18+8 \cdot\left(\frac{3}{2}\right)^{\alpha}+2 \cdot 3^{\alpha}\right)}+\frac{2 \cdot\left(\frac{3}{2}\right)^{\alpha}\left(f_{2}-f_{-2}\right)}{h\left(18+8 \cdot\left(\frac{3}{2}\right)^{\alpha}+2 \cdot 3^{\alpha}\right)} \\
& +\frac{3\left(f_{3}-f_{-3}\right)}{h\left(18+8 \cdot\left(\frac{3}{2}\right)^{\alpha}+2 \cdot 3^{\alpha}\right)}
\end{aligned}
$$

Here, $f_{j}:=f\left(x_{j}\right)$.

Proof. Since nothing has to be done as a little bit of arithmetic there is nothing to prove. Notice that $w^{(-j)}\left(x_{0}\right)=w^{(j)}\left(x_{0}\right)$ and $w^{(i)}\left(x_{0}\right)=1 /(i \cdot h)^{\alpha}$ has to be used.

In order to look more closely into these formulae we exploit Taylor series in the form of

$$
f_{j}=f\left(x_{0}\right)+j \cdot h \cdot f^{\prime}\left(x_{0}\right)+\frac{(j \cdot h)^{2}}{2 !}+\mathcal{O}\left(h^{3}\right)
$$

which we insert into (15), (16), (17). This gives, after some algebra,

Lemma 5.2. The difference formulae (15)-(17) are consistent approximations to the first derivative of $f$. In particular, (15) yields

$$
\frac{\mathrm{d} G f}{\mathrm{~d} x}\left(x_{0}\right)=\frac{\mathrm{d} f}{\mathrm{~d} x}\left(x_{0}\right)+\underbrace{\frac{h^{2}}{3 !}}_{=: L_{1} h^{2}} f^{\prime \prime \prime}\left(x_{0}\right)+\mathcal{O}\left(h^{4}\right)
$$

(16) leads to

$$
\frac{\mathrm{d} G f}{\mathrm{~d} x}\left(x_{0}\right)=\frac{\mathrm{d} f}{\mathrm{~d} x}\left(x_{0}\right)+\underbrace{\frac{\left(16+2^{\alpha}\right) h^{2}}{3 !\left(2^{\alpha}+4\right)}}_{=: L_{2} h^{2}} f^{\prime \prime \prime}\left(x_{0}\right)+\mathcal{O}\left(h^{4}\right)
$$




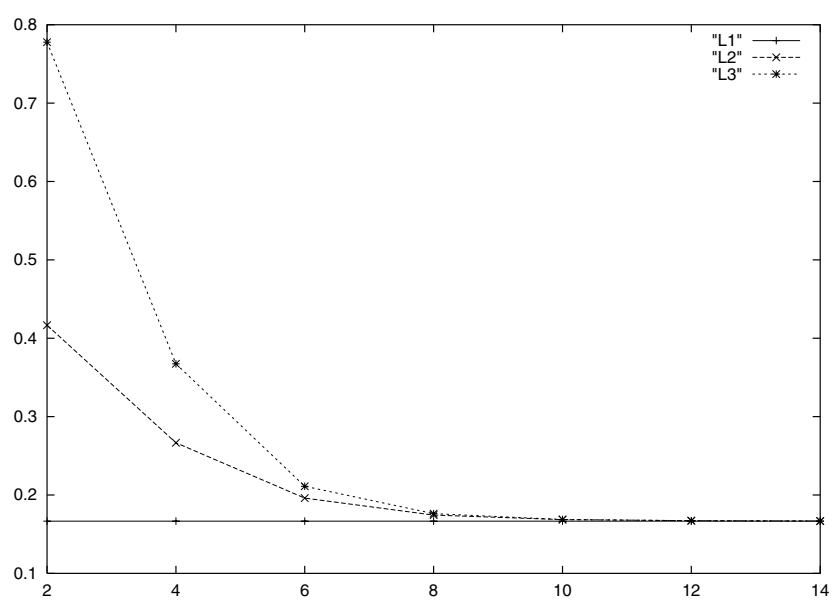

FIGURE 10. Size of error term $L_{i}$ plotted versus $\alpha$.

and from (17) we get

$$
\frac{\mathrm{d} G f}{\mathrm{~d} x}\left(x_{0}\right)=\frac{\mathrm{d} f}{\mathrm{~d} x}\left(x_{0}\right)+\underbrace{\frac{\left(27+\frac{16}{3}\left(\frac{3}{2}\right)^{\alpha}+3^{\alpha-1}\right) h^{2}}{18+8\left(\frac{3}{2}\right)^{\alpha}+2 \cdot 3^{\alpha}}}_{=: L_{3} h^{2}} f^{\prime \prime \prime}\left(x_{0}\right)+\mathcal{O}\left(h^{4}\right) .
$$

In comparing the $\alpha$-depending factors $L_{i}, i=1,2,3$, which determine the error level (not the order of error) we see in Figure 10 that the quality of the numerical derivative deteriorates with the number of points. While the three-point formula is exactly the optimal formula the truncation error is higher for small values of $\alpha$ and approaches the optimal value of $L_{1}=1 / 6$ from $\alpha=10 \mathrm{on}$. Hence, for a classical IMLS method based on a linear basis it seems always advisable to choose large weights in order to reduce the error in the first derivative. We now turn our attention to the IMLS interpolant based on the quadratic basis and inspect the first derivative

$$
\frac{\mathrm{d} G f}{\mathrm{~d} x}\left(x_{k}\right)=\beta_{2}\left(x_{k}\right)+2 x_{k} \beta_{3}\left(x_{k}\right)
$$

as derived in Lemma 4.6 .

Lemma 5.3. In case of a three-point grid the first derivative of the "quadratic" IMLS interpolant gives the finite difference formula

$$
\frac{\mathrm{d} G f}{\mathrm{~d} x}\left(x_{0}\right)=\frac{f_{1}-f_{-1}}{2 h},
$$

while the difference formula for a five-point grid is

$$
\frac{\mathrm{d} G f}{\mathrm{~d} x}\left(x_{0}\right)=\frac{1}{\mathfrak{a} h}\left(\mathfrak{c}_{2} f_{2}+\mathfrak{c}_{1} f_{1}-\mathfrak{c}_{1} f_{-1}-\mathfrak{c}_{2} f_{-2}\right)
$$

with

$$
\begin{aligned}
\mathfrak{a} & =2\left(64+5 \cdot 2^{2+\alpha}+4^{\alpha}\right) \\
\mathfrak{c}_{2} & =32+2^{1+\alpha} \\
\mathfrak{c}_{1} & =2^{4+\alpha}+4^{\alpha} .
\end{aligned}
$$


On a seven-point grid the difference formula turns out to read as

$$
\frac{\mathrm{d} G f}{\mathrm{~d} x}\left(x_{0}\right)=\frac{1}{\mathfrak{a} h}\left(\mathfrak{c}_{3} f_{3}+\mathfrak{c}_{2} f_{2}+\mathfrak{c}_{1} f_{1}-\mathfrak{c}_{1} f_{-1}-\mathfrak{c}_{2} f_{-2}-\mathfrak{c}_{3} f_{-3}\right)
$$

with

$$
\begin{aligned}
\mathfrak{a} & =2\left(5 \cdot 2^{1+2 \alpha} \cdot 3^{2+\alpha}+729 \cdot 4^{\alpha}+13 \cdot 6^{2+\alpha}+64 \cdot 9^{\alpha}+5 \cdot 2^{2+\alpha} \cdot 9^{\alpha}+36^{\alpha}\right) \\
\mathfrak{c}_{3} & =2^{4+\alpha} \cdot 3^{1+\alpha}+243 \cdot 4^{\alpha}+3^{1+\alpha} \cdot 4^{\alpha} \\
\mathfrak{c}_{2} & =2\left(2^{\alpha} \cdot 3^{4+\alpha}+16 \cdot 9^{\alpha}+18^{\alpha}\right) \\
\mathfrak{c}_{1} & =3^{4+\alpha} \cdot 4^{\alpha}+2^{4+\alpha} \cdot 9^{\alpha}+36^{\alpha} .
\end{aligned}
$$

Proof. The proof follows from an enormous amount of elementary algebra and noting that $w^{(-i)}\left(x_{0}\right)=w^{(i)}\left(x_{0}\right)$, $w^{(i)}\left(x_{0}\right)=(i \cdot h)^{-\alpha}$. While the five-point formula can be computed by hand Mathematica was used to compute the coefficients of the seven-point formula.

As in the case of the IMLS interpolant based on the linear basis we end with a remark on the consistency of the above difference formulae.

Lemma 5.4. The difference formulae (18)-(20) are consistent approximations to the first derivative of $f$. In particular, (18) yields

(19) leads to

$$
\frac{\mathrm{d} G f}{\mathrm{~d} x}\left(x_{0}\right)=\frac{\mathrm{d} f}{\mathrm{~d} x}\left(x_{0}\right)+\underbrace{\frac{h^{2}}{3 !}}_{=: Q_{1} h^{2}} f^{\prime \prime \prime}\left(x_{0}\right)+\mathcal{O}\left(h^{4}\right),
$$

$$
\begin{aligned}
\frac{\mathrm{d} G f}{\mathrm{~d} x}\left(x_{0}\right)= & \frac{\mathrm{d} f}{\mathrm{~d} x}\left(x_{0}\right)+\underbrace{\frac{\left(16+2^{\alpha}\right)^{2} h^{2}}{6\left(64+5 \cdot 2^{2+\alpha}+4^{\alpha}\right)}}_{=: Q_{2} h^{2}} f^{\prime \prime \prime}\left(x_{0}\right) \\
& +\frac{\left(1024+5 \cdot 2^{4+\alpha}+4^{\alpha}\right) h^{4}}{120\left(64+5 \cdot 2^{2+\alpha}+4^{\alpha}\right)} f^{(v)}\left(x_{0}\right)+\mathcal{O}\left(h^{6}\right),
\end{aligned}
$$

and from (20) we get

$$
\begin{aligned}
\frac{\mathrm{d} G f}{\mathrm{~d} x}\left(x_{0}\right)=\frac{\mathrm{d} f}{\mathrm{~d} x}\left(x_{0}\right)+\underbrace{\frac{\left(2^{5+\alpha} \cdot 3^{4+\alpha}+2^{1+2 \alpha} \cdot 3^{4+\alpha}+6561 \cdot 4^{\alpha}+256 \cdot 9^{\alpha}+2^{5+\alpha} \cdot 9^{\alpha}+36^{\alpha}\right) h^{2}}{6\left(5 \cdot 2^{1+2 \alpha} \cdot 3^{2+\alpha}+729 \cdot 4^{\alpha}+13 \cdot 6^{2+\alpha}+64 \cdot 9^{\alpha}+5 \cdot 2^{2+\alpha} \cdot 9^{\alpha}+36^{\alpha}\right)}}_{=: Q_{3} h^{2}} f^{\prime \prime \prime}\left(x_{0}\right) \\
+\frac{\left(5 \cdot 2^{1+2 \alpha} \cdot 3^{4+\alpha}+59049 \cdot 4^{\alpha}+13 \cdot 6^{4+\alpha}+1024 \cdot 9^{\alpha}+5 \cdot 2^{4+\alpha} \cdot 9^{\alpha}+36^{\alpha}\right) h^{4}}{120\left(5 \cdot 2^{1+2 \alpha} \cdot 3^{2+\alpha}+729 \cdot 4^{\alpha}+13 \cdot 6^{2+\alpha}+64 \cdot 9^{\alpha}+5 \cdot 2^{2+\alpha} \cdot 9^{\alpha}+36^{\alpha}\right)} f^{(v)}\left(x_{0}\right)+\mathcal{O}\left(h^{5}\right) .
\end{aligned}
$$

Proof. The proof follows from inserting Taylor series expansions into the difference formulae of Lemma 5.3.

Although clearly $Q_{1} \equiv L_{1}$ one could conclude that since the construction of the "quadratic" IMLS interpolant includes the "linear" interpolant by construction we may also conjecture that $Q_{2}$ and $Q_{3}$ are in fact identical to the corresponding error terms in the "linear" interpolant. In fact, this conclusion is true.

Lemma 5.5. The error terms $Q_{1}, Q_{2}, Q_{3}$ corresponding to the order $h^{2}$ are identical to the error terms in the IMLS interpolant based on the linear basis, i.e.

$$
Q_{i} \equiv L_{i}, \quad i=1,2,3 .
$$

Proof. Use simple algebra to transform $Q_{i}$ into $L_{i}$. 


\subsection{Differences at the boundary}

The differences studied so far are central differences. At the boundaries of a domain or in an upwind application to transport equations of mathematical physics one-sided differences are needed. It is obvious that one-sided differences involving the same number of points as corresponding central differences suffer from larger errors. In the case of IMLS interpolants we prove

Lemma 5.6. Let $x_{0}$ be a left boundary point and consider a two-point configuration $x_{0}, x_{1}$ where $x_{i}=x_{0}+h$. In the case of the IMLS interpolant based on the linear polynomial basis the resulting one-sided finite difference is given by

$$
D G f\left(x_{0}\right)=\frac{f_{1}-f_{0}}{h}
$$

In a three-point configuration $x_{0}, x_{1}, x_{2}$ where $x_{i}=x_{0}+i \cdot h, i=1,2$ the resulting difference is

$$
\operatorname{DGf}\left(x_{0}\right)=\frac{2^{1-\alpha} f_{2}+f_{1}-\left(1+2^{1-\alpha}\right) f_{0}}{\left(1+2^{2-\alpha}\right) h} .
$$

This difference is a consistent approximation to $f^{\prime}$ at $x_{0}$ and it holds

$$
D G f\left(x_{0}\right)=f^{\prime}\left(x_{0}\right)+\frac{\left(8+2^{\alpha}\right)\left(x_{0} f^{\prime \prime}\left(x_{0}\right)-f^{\prime}\left(x_{0}\right)\right)}{2\left(4+2^{\alpha}\right) x_{0}} h+\mathcal{O}\left(h^{2}\right) .
$$

Proof. Use again the formulae derived for the derivative and then insert Taylor series.

Comparing the above finite difference with the optimal differences listed in [4] we may note some facts. The optimal one sided differences are

$$
D f\left(x_{0}\right)=\frac{f_{1}-f_{0}}{h}
$$

which is a first order approximation as in (21) and (22) but uses only two points, or

$$
D f\left(x_{0}\right)=\frac{-\frac{1}{2} f_{2}-\frac{3}{2} f_{1}+2 f_{0}}{h}
$$

which is of second order but uses three points. In the case of (23) a Taylor series analysis reveals

$$
D f\left(x_{0}\right)=f^{\prime}\left(x_{0}\right)+\frac{h}{2} f^{\prime \prime}\left(x_{0}\right)+\mathcal{O}\left(h^{2}\right)
$$

while in the case of difference (24) we get

$$
D f\left(x_{0}\right)=f^{\prime}\left(x_{0}\right)-\frac{h^{2}}{3} f^{\prime \prime \prime}\left(x_{0}\right)+\mathcal{O}\left(h^{3}\right) .
$$

Hence, the IMLS interpolant based on the linear basis gives the optimal formula for the first derivative on two points at the boundary. The difference formula resting on three grid points is sub-optimal. 


\subsection{Second derivatives}

As was already shown before the IMLS interpolant based on the linear polynomial basis results in an inconsistent second derivative. Hence, we are only interested in the second derivative stemming from the "quadratic" interpolant. We use Lemma 4.6 and compute $\frac{\mathrm{d} \beta_{2}}{\mathrm{~d} x}\left(x_{0}\right), \beta_{3}\left(x_{0}\right)$, and $\frac{\mathrm{d} \beta_{3}}{\mathrm{~d} x}\left(x_{0}\right)$ on a three-point grid $x_{-1}, x_{0}, x_{1}$.

Lemma 5.7. The IMLS interpolant based on linear polynomials results on a three-node grid in the finite difference

$$
\frac{D^{2} G f}{\mathrm{~d} x^{2}}\left(x_{0}\right)=\frac{f_{1}-2 f_{0}+f_{-1}}{h^{2}} .
$$

If $x_{0}$ is a boundary point, then the three-point one-sided difference is

$$
\frac{D^{2} G f}{\mathrm{~d} x^{2}}\left(x_{0}\right)=\frac{f_{0}-2 f_{1}+f_{2}}{h^{2}}
$$

In case of four points $x_{0}, x_{1}, x_{2}, x_{3}$ at the boundary the IMLS interpolant yields

$$
\frac{D^{2} G f}{\mathrm{~d} x^{2}}\left(x_{0}\right)=\frac{\mathfrak{c}_{\mathfrak{o}} f_{0}-\mathfrak{c}_{1} f_{1}+\mathfrak{c}_{2} f_{2}+\mathfrak{c}_{3} f_{3}}{\mathfrak{c} h^{2}}
$$

where

$$
\begin{aligned}
\mathfrak{c} & =2^{\alpha} \cdot 3^{2+\alpha}+3^{2+\alpha} \cdot 4^{\alpha}+18^{\alpha} \\
\mathfrak{c}_{0} & =13 \cdot 2^{\alpha} \cdot 3^{1-\alpha}+2^{1+2 \alpha} \cdot 3^{1+\alpha}-6^{2+\alpha}+18^{\alpha} \\
\mathfrak{c}_{1} & =3^{2+\alpha} \cdot 4^{\alpha}+2^{1+\alpha} \cdot 9^{\alpha} \\
\mathfrak{c}_{2} & =18^{\alpha}-2^{\alpha} \cdot 3^{2+\alpha} \\
\mathfrak{c}_{3} & =3^{1+\alpha} \cdot 4^{\alpha}+6^{1+\alpha} .
\end{aligned}
$$

Concerning the order of accuracy formula (25) yields

$$
\frac{D^{2} G f}{\mathrm{~d} x^{2}}\left(x_{0}\right)=f^{\prime \prime}\left(x_{0}\right)+\frac{1}{12} f^{(i v)}\left(x_{0}\right) h^{2}+\mathcal{O}\left(h^{3}\right),
$$

formula (26) gives

$$
\frac{D^{2} G f}{\mathrm{~d} x^{2}}\left(x_{0}\right)=f^{\prime \prime}\left(x_{0}\right)+f^{\prime \prime \prime}\left(x_{0}\right) h+\mathcal{O}\left(h^{2}\right),
$$

and formula (27) leads to

$$
\begin{aligned}
\frac{D^{2} G f}{\mathrm{~d} x^{2}}\left(x_{0}\right)= & f^{\prime \prime}\left(x_{0}\right)+\frac{\left(5 \cdot 2^{\alpha} \cdot 3^{1+\alpha}+12^{1+\alpha}+18^{\alpha}\right) f^{\prime \prime \prime}\left(x_{0}\right)}{2^{\alpha} \cdot 3^{2+\alpha}+3^{2+\alpha} \cdot 4^{\alpha}+18^{\alpha}} h \\
& \frac{\left(19 \cdot 2^{\alpha} \cdot 3^{2+\alpha}+13 \cdot 3^{2+\alpha} \cdot 4^{\alpha}+7 \cdot 18^{\alpha}\right) f^{(i v)}\left(x_{0}\right)}{12\left(2^{\alpha} \cdot 3^{2+\alpha}+3^{2+\alpha} \cdot 4^{\alpha}+18^{\alpha}\right)} h^{2}+\mathcal{O}\left(h^{3}\right) .
\end{aligned}
$$

Proof. Use the formulae for the derivatives and Taylor series expansions. 


\section{Conclusions}

We have established a link between the first and second derivatives of IMLS interpolants based on a linear and quadratic polynomial basis, respectively, and finite difference operators. It was shown that the higher derivatives of the Shepard interpolant vanish at the nodes and how this fact could be used to derive exact formulae for the IMLS derivatives. Furthermore, our analysis revealed explicit finite difference operators which correspond to the derivatives of the interpolant and that optimal differences are in fact recovered if the support of the weight function is suitably restricted. Although it is clear the the order of approximation of difference operators can not be increased by enlarging the support of the IMLS weights we compute exactly the leading error terms and their dependence on the weights. However, open problems remain. With the given analysis on three, five and seven points we could not explain the loss of consistency encountered in the second derivative of the quadratic interpolant. It seems very likely that global tools of analysis have to be used to answer this problem conclusively instead of the local tool of finite difference operators.

Acknowledgements. The author acknowledges the support of the Deutsche Forschungsgemeinschaft DFG via the project So 363/8-1 which was stopped too early.

The constructive remarks of an unknown referee helped to shape even the details of the manuscript. This help is gratefully acknowledged.

\section{REFERENCES}

[1] T. Belytschko, Y. Krongauz, D. Organ, M. Fleming and P. Krysl, Meshless methods: an overview and recent developments. Comput. Methods Appl. Mech. Engrg. 139 (1996) 3-47.

[2] J.P. Boyd, Chebyshev and Fourier Spectral Methods. Springer Verlag (1989).

[3] B. Fornberg, Generation of Finite Difference Formulas on Arbitrarily Spaced Grids. Math. Comp. 51 (1988) $699-706$.

[4] B. Fornberg, A Practical Guide to Pseudospectral Methods. Cambridge University Press (1996).

[5] J. Fürst and Th. Sonar, On meshless collocation approximations of conservation laws: preliminary investigations on positive schemes and dissipation models. ZAMM Z. Angew. Math. Mech. 81 (2001) 403-415.

[6] M. Kunle, Entwicklung und Untersuchung von Moving Least Square Verfahren zur numerischen Simulation hydrodynamischer Gleichungen. Doktorarbeit, Fakultät für Physik, Eberhard-Karls-Universität zu Tübingen (2001).

[7] P. Lancaster and K. Šalkauskas, Surfaces generated by moving least squares methods. Math. Comp. 37 (1981) $141-158$.

[8] P. Lancaster and K. Šalkauskas, Curve and Surface Fitting: An Introduction. Academic Press (1986).

[9] T. Liszka and J. Orkisz, The finite difference method at arbitrary irregular grids and its application in applied mechanics. Comput. Structures 11 (1980) 83-95.

[10] H. Netuzylov, Th. Sonar and W. Yomsatieankul, Finite difference operators from moving least squares interpolation. Manuscript, Institut Computational Mathematics, TU Braunschweig (2004).

[11] N. Perrone and R. Kao, A general finite difference method for arbitrary meshes. Comput. Structures 5 (1975) 45-58.

[12] W. Schönauer, Generation of difference and error formulae of arbitrary consistency order on an unstructured grid. ZAMM Z. Angew. Math. Mech. 78 (1998) S1061-S1062.

[13] L. Theilemann, Ein gitterfreies differenzenverfahren. Doktorarbeit, Institut für Aerodynamik und Gasdynamik, Universität Stuttgart (1983).

[14] W. Yomsatieankul, Th. Sonar and H. Netuzhylov, Spatial difference operators from moving least squares interpolation. Manuscript, Institut Computational Mathematics, TU Braunschweig (2004). 